\title{
A Fixed Point Technique for Set-Valued Contractions with Supportive Applications
}

\author{
Hasanen A. Hammad ${ }^{1}{ }^{1}$ and Manuel De la Sen $\mathbb{D D}^{2}$ \\ ${ }^{1}$ Department of Mathematics, Faculty of Science, Sohag University, Sohag 82524, Egypt \\ ${ }^{2}$ Institute of Research and Development of Processes, University of the Basque Country, 48940 Leioa Bizkaia, Spain
}

Correspondence should be addressed to Hasanen A. Hammad; h.elmagd89@gmail.com

Received 12 April 2021; Revised 16 June 2021; Accepted 26 June 2021; Published 12 July 2021

Academic Editor: Maria L. Gandarias

Copyright (c) 2021 Hasanen A. Hammad and Manuel De la Sen. This is an open access article distributed under the Creative Commons Attribution License, which permits unrestricted use, distribution, and reproduction in any medium, provided the original work is properly cited.

In this manuscript, exciting fixed point results for a pair of multivalued mappings justifying rational Gupta-Saxena type $\Omega$-contractions in the setting of extended $b$-metric-like spaces are established. The theoretical results have also been strengthened by some nontrivial examples. Finally, the theoretical results are used to study the existence of the solution of Fredholm integral equation which arises from the damped harmonic oscillator, to study initial value problem which arises from Newton's law of cooling and to study infinite systems of fractional ordinary differential equations (ODEs).

\section{Introduction}

The importance of fixed-point (FP) theory increased after Banach introduced his principle [1]. It became an essential tool in nonlinear analysis. In particular, there are many important applications in which the FP method is involved such as economics, microbiology, and physical applications. This technique permeates fractional analysis and becomes a basic method in finding solutions to some fractional systems. For more details, we suggest the reader to read the books of Kilbas et al. [2], Samko et al. [3], Wang et al. [4], and Atangana and Baleanu [5]. Furthermore, this methodology is widely used in finding solutions of integral equations, fractional differential equations, and boundary value problems, for example, see [6-11].

Under Hausdorff metric space formalism, Nadler [12] introduced the notion of multivalued mapping as follows: Let $(V, \tau)$ be a metric space, $V_{\mathrm{CB}}$ represents the set of nonempty closed bounded subsets of $V$, and let $\Xi: V_{\mathrm{CB}} \times$ $V_{\mathrm{CB}} \longrightarrow \mathbb{R}^{+}$be a function given by

$$
\Xi\left(z_{1}, z_{2}\right)=\max \left\{\sup _{\xi \in z_{1}} \tau\left(\xi, z_{2}\right), \sup _{\zeta \in z_{2}} \tau\left(\zeta, z_{1}\right)\right\}, \forall z_{1}, z_{2} \in V_{\mathrm{CB}}
$$

where $\tau\left(\xi, \mho_{2}\right)=\inf \left\{\tau(\xi, \zeta): \zeta \in z_{2}\right\}$.
The function $\Xi$ is called also the Hausdorff-Pompeiu metric. Consider

$$
\delta\left(z_{1}, z_{2}\right)=\sup \left\{\tau(\xi, \zeta): \xi \in z_{1}, \zeta \in z_{2}\right\}
$$

then, one can deduce that for all $z_{1}, z_{2}, z_{3} \in V_{\mathrm{CB}}$,

(i) $\delta\left(z_{1}, z_{2}\right)=\delta\left(z_{2}, z_{1}\right)$

(ii) $\delta\left(z_{1}, z_{2}\right)=0 \Leftrightarrow z_{1}=z_{2}=\{\xi\}$

(iii) $\delta\left(z_{1}, z_{3}\right) \leq \delta\left(z_{1}, z_{2}\right)+\delta\left(z_{2}, z_{3}\right)$

(iv) $\delta\left(z_{1}, z_{1}\right)=d i a m z_{1}$

In 2012, Wardowski [13] generalized the old principle of Banach to a broader kind. He called it $\Omega$-contraction and presented it in the following definition:

Definition 1. A nonlinear mapping $N: V \longrightarrow V$ described on the metric space $(V, \tau)$ is called a $\Omega$-contraction if there exist $F \in \Sigma$ and $v>0$ so that

$$
\tau(N \wp, N \hbar)>0 \Rightarrow v+\Omega(\tau(N \wp, N \hbar)) \leq \Omega(\tau(\wp, \hbar)), \forall \wp, \hbar \in V,
$$


where $\Sigma$ is the class of functions $\Omega:(0,+\infty) \longrightarrow \mathbb{R}$ so that the assertions below hold:

$\left(\Omega_{1}\right)$ for all $z_{1}, z_{1} \in \mathbb{R}^{+}$, if $z_{1}<z_{2}$, then $\Omega\left(z_{1}\right)<\Omega\left(z_{2}\right)$

$\left(\Omega_{2}\right)$ for all positive real sequence $\left\{z_{r}\right\}, z_{r} \longrightarrow 0$ if and only if $\Omega\left(z_{r}\right) \longrightarrow-\infty$ as $r \longrightarrow \infty$

$\left(\Omega_{3}\right)$ there exists $\ell \in(0,1)$ so that $\lim _{s \longrightarrow 0^{+}} s_{\ell} \Omega(s)=0$

Many researchers have turned to generalize ordinary metric spaces to more comprehensive spaces such as $b$-metric, metric-like, and $b$-metric-like spaces. In the other word, Czerwik [14] was able to present $b$-metric spaces, Alghmandi et al. [15] introduced metric-like and $b$-metric-like spaces, and Hammad et al. [16] presented extended $b$-metric like spaces. It should be noted that the definitions of these spaces are mentioned in the next section.

The concept of multivalued mapping and $\Omega$-contraction mapping in abstract spaces are combined, and fruitful FP results in this line are obtained. For instance, see Cosentino and Vetro [17], Sgroi and Vetro [18], and Ali et al. [19].

By the same approach, in this manuscript, some FP results under rational Gupta-Saxena type $\Omega$-contractions (for short, $\Phi_{\varphi, \Omega}^{r G S}$-contractions) are obtained in the setting of extended $b$-metric-like space (for short, $¥_{b}$-spaces). Also, the results are supported by nontrivial examples. Finally, the theoretical results are applied to study the existence of the solution to the Fredholm integral equation which arises from the damped harmonic oscillator, to study the initial value problem (IVP) which arises from Newton's law of cooling and to study infinite systems of fractional ODEs.

\section{Preliminaries}

This section discusses some extended spaces and their topological properties.

Definition 2 [15]. Let $V$ be a nonempty set. A function $\tau$ : $V^{2} \longrightarrow \mathbb{R}^{+}$is called a metric-like on $V$, if for all $z_{1}, z_{2}, z_{3} \in$ $V$, the conditions below hold:

(a) $\tau\left(z_{1}, z_{2}\right)=0 \Rightarrow z_{1}=z_{2}$

(b) $\tau\left(z_{1}, z_{2}\right)=\tau\left(z_{2}, z_{1}\right)$

(c) $\tau\left(z_{1}, z_{3}\right) \leq \tau\left(z_{1}, z_{2}\right)+\tau\left(z_{2}, z_{3}\right)$

Here, $(V, \tau)$ is named as a metric-like space.

Definition 3 [15]. A $b$-metric-like on a nonempty set $V$ is a function $\tau: V \times V \longrightarrow \mathbb{R}^{+}$so that for all $z_{1}, z_{2}, z_{3} \in V$ and a constant $j \geq 1$, the following three conditions hold:

(e) $\tau\left(z_{1}, z_{2}\right)=0 \Rightarrow z_{1}=z_{2}$

(f) $\tau\left(z_{1}, z_{2}\right)=\tau\left(z_{2}, z_{1}\right)$

(g) $\tau\left(z_{1}, z_{3}\right) \leq j\left[\tau\left(z_{1}, z_{2}\right)+\tau\left(z_{2}, z_{3}\right)\right]$

Here, $(V, \tau)$ is called a $b$-metric-like space with constant $j$.
For more details about the conversion of metric-like and $b$-metric-like spaces, see [20-22].

Very recently, Hammad et al. [16] merged the results of metric-like with $b$-metric-like spaces and formed a $¥_{b}$-space. Also, topological properties of this space were discussed, and some nice FP results of Wardowski and Suzuki contraction mappings were obtained. This space is introduced as follows:

Definition 4 [16]. Assume that $V$ is a nonempty set and $j$ : $V \times V \longrightarrow[1, \infty)$ is a given function. A function $\tau_{j}: V^{2}$ $\longrightarrow[0, \infty)$ is called a modified $\tau_{j}$-metric-like if for all $z_{1}$, $z_{2}, z_{3} \in V$ :

$\left(\tau_{j 1}\right) \tau_{j}\left(z_{1}, z_{2}\right)=0 \Rightarrow z_{1}=z_{2}$

$\left(\tau_{j 2}\right) \tau_{j}\left(z_{1}, z_{2}\right)=\tau_{j}\left(z_{2}, z_{1}\right)$

$\left(\tau_{j 3}\right) \tau_{j}\left(z_{1}, z_{3}\right) \leq j\left(z_{1}, z_{3}\right)\left[\tau_{j}\left(z_{1}, z_{2}\right)+\tau_{j}\left(z_{2}, z_{3}\right)\right]$

Then, $\left(V, \tau_{j}\right)$ is called a $¥_{b}$-space.

Note that the class of $¥_{b}$-spaces is larger than the class of $b$-metric-like spaces by replacing the constant $j \geq 1$ of Definition 3 by a nonconstant function $j: V \times V \longrightarrow[1, \infty)$ of Definition 4.

For more examples and applications via the mentioned space, see [16].

Definition 5 [16]. Let $\left\{z_{l}\right\}$ be a sequence in the $¥_{b}$-space $\left(V, \tau_{j}\right)$.

(i) If $\lim _{i \longrightarrow \infty} \tau_{j}\left(z_{i}, z\right)=\tau_{j}(z, z)$, then $\left\{z_{i}\right\}$ is convergent to $z$

(ii) $\left\{z_{i}\right\}$ is called Cauchy if $\lim _{i, k \longrightarrow \infty} \tau_{j}\left(z_{i}, z_{k}\right)$ exists and is finite

(iii) If for each Cauchy sequence $\left\{z_{i}\right\}$, there is $z \in V$, so that $\lim _{i, k \longrightarrow \infty} \tau_{j}\left(z_{i}, z_{k}\right)=\tau_{j}(z, z)=\lim _{\iota \longrightarrow \infty} \tau_{j}\left(z_{i}, z\right)$; then, $\left(V, \tau_{j}\right)$ is called complete

Lemma 6 [4]. Suppose that $(V, \tau)$ is an extended b-metric space and that $V_{1}, V_{2} \in V_{C B}, \mu \in V_{1}$ and $\lambda>1$, there exists $\eta(\mu) \in V_{2}$ so that $\omega_{\theta}(\mu, \eta) \leq \lambda \Xi\left(V_{1}, V_{2}\right)$.

It should be noted that Lemma 6 also satisfied in each of metric spaces [23] and $b$-metric spaces [24]. Hence, it can be easily achieved in $¥_{b}$-spaces.

\section{Main Theorem}

We begin this part with the following definition:

Definition 7. Let $\left(V, \tau_{j}\right)$ be $\dddot{\#}_{b}$-space with a function $j: V \times$ $V \longrightarrow[1, \infty)$. Assume that $\Phi, \varphi: V \longrightarrow V_{C B}$ are two multivalued mappings. We say that the pair $(\Phi, \varphi)$ is a $\Phi_{\varphi, \Omega^{-}}^{r G S}$ contraction if there exist $v>0$ and $\Omega \in \Sigma$ so that for all $z_{1}, z_{2} \in V$, then

$$
v+\Omega\left(\lambda H\left(\Phi z_{1}, \varphi z_{2}\right)\right) \leq \Omega\left(\beta\left(z_{1}, z_{2}\right)\right)
$$


where

$$
\begin{aligned}
& \beta\left(z_{1}, z_{2}\right) \\
& \quad=\max \left\{\tau_{j}\left(z_{1}, z_{2}\right), \frac{\left[1+\tau_{j}\left(z_{1}, \Phi z_{1}\right)\right] \tau_{j}\left(z_{2}, \varphi z_{2}\right)}{1+\tau_{j}\left(z_{1}, z_{2}\right)}, \frac{\tau_{j}\left(z_{1}, \Phi z_{1}\right) \tau_{j}\left(z_{2}, \varphi z_{2}\right)}{1+\tau_{j}\left(z_{1}, z_{2}\right)}\right\}
\end{aligned}
$$

Theorem 8. Let $\left(V, \tau_{j}\right)$ be a complete $¥_{b}$-space and let $(\varphi$, $\Phi)$ be a $\Phi_{\varphi, \Omega}^{r G S}$-contraction so that for all $z_{0} \in V$, $\lim _{m . n \longrightarrow \infty} j\left(z_{m}, z_{n}\right)<1 / \lambda$. Then, $\varphi$ and $\Phi$ have a unique common FP.
Proof. Let $z_{0} \in V$ and $z_{1} \in \Phi z_{0}$. Assume that $z_{2}=\varphi z_{1}$, and by Lemma 6 , there is $z_{3} \in \Phi z_{2}$ so that

$$
\tau_{j}\left(z_{3}, z_{2}\right) \leq \lambda H\left(\Phi z_{2}, \varphi z_{1}\right)
$$

Inductively, we let $z_{2 n}=\varphi z_{2 n-1}$, and by Lemma 6 , we select $z_{2 n+1} \in \Phi z_{2 n}$ so that

$$
\tau_{j}\left(z_{2 n-1}, z_{2 n}\right) \leq \lambda H\left(\Phi z_{2 n}, \varphi z_{2 n-1}\right) .
$$

Applying (4) and (7), we have

$$
\begin{aligned}
v+\Omega\left(\tau_{j}\left(z_{2 n+1}, z_{2 n+2}\right)\right) & \leq v+\Omega\left(\lambda H\left(\Phi z_{2 n}, \varphi z_{2 n+1}\right)\right) \\
& \leq \Omega\left(\max \left\{\tau_{j}\left(z_{2 n}, z_{2 n+1}\right), \frac{\left[1+\tau_{j}\left(z_{2 n}, \Phi z_{2 n}\right)\right] \tau_{j}\left(z_{2 n+1}, \varphi z_{2 n+1}\right)}{1+\tau_{j}\left(z_{2 n}, z_{2 n+1}\right)}, \frac{\tau_{j}\left(z_{2 n}, \Phi z_{2 n}\right) \tau_{j}\left(z_{2 n+1}, \varphi z_{2 n+1}\right)}{1+\tau_{j}\left(z_{2 n}, z_{2 n+1}\right)}\right\}\right) \\
& =\Omega\left(\max \left\{\tau_{j}\left(z_{2 n}, z_{2 n+1}\right), \frac{\left[1+\tau_{j}\left(z_{2 n}, z_{2 n+1}\right)\right] \tau_{j}\left(z_{2 n+1}, z_{2 n+2}\right)}{1+\tau_{j}\left(z_{2 n}, z_{2 n+1}\right)}, \frac{\tau_{j}\left(z_{2 n}, z_{2 n+1}\right) \tau_{j}\left(z_{2 n+1}, z_{2 n+2}\right)}{1+\tau_{j}\left(z_{2 n}, z_{2 n+1}\right)}\right\}\right) \\
& \leq \Omega\left(\max \left\{\tau_{j}\left(z_{2 n}, z_{2 n+1}\right), \tau_{j}\left(z_{2 n+1}, z_{2 n+2}\right), \tau_{j}\left(z_{2 n+1}, z_{2 n+2}\right)\right\}\right) \\
& =\Omega\left(\max \left\{\tau_{j}\left(z_{2 n}, z_{2 n+1}\right), \tau_{j}\left(z_{2 n+1}, z_{2 n+2}\right)\right\}\right) .
\end{aligned}
$$

If $\max \left\{\tau_{j}\left(z_{2 n}, z_{2 n+1}\right), \tau_{j}\left(z_{2 n+1}, z_{2 n+2}\right)\right\}=\tau_{j}\left(z_{2 n+1}, z_{2 n+2}\right)$, then

$$
v+\Omega\left(\tau_{j}\left(z_{2 n+1}, z_{2 n+2}\right)\right) \leq \Omega\left(\tau_{j}\left(z_{2 n+1}, z_{2 n+2}\right)\right) .
$$

This is a contradiction according to $\Omega_{1}$. Therefore, $\max \left\{\tau_{j}\left(z_{2 n}, z_{2 n+1}\right), \tau_{j}\left(z_{2 n+1}, z_{2 n+2}\right)\right\}=\tau_{j}\left(z_{2 n}, z_{2 n+1}\right)$. Then, from $(8)$,

$$
\Omega\left(\tau_{j}\left(z_{2 n+1}, z_{2 n+2}\right)\right) \leq \Omega\left(\tau_{j}\left(z_{2 n}, z_{2 n+1}\right)\right)-v, \forall n \geq 0 .
$$

Similarly, we get

$$
\Omega\left(\tau_{j}\left(z_{2 n}, z_{2 n+1}\right)\right) \leq \Omega\left(\tau_{j}\left(z_{2 n-1}, z_{2 n}\right)\right)-v .
$$

Applying (10) and (11), we have

$$
\Omega\left(\tau_{j}\left(z_{2 n+1}, z_{2 n+2}\right)\right) \leq \Omega\left(\tau_{j}\left(z_{2 n-1}, z_{2 n}\right)\right)-2 v .
$$

Repeating consequently, one can obtain

$$
\Omega\left(\tau_{j}\left(z_{2 n+1}, z_{2 n+2}\right)\right) \leq \Omega\left(\tau_{j}\left(z_{0}, z_{1}\right)\right)-(2 n+1) v .
$$

Similarly,

$$
\Omega\left(\tau_{j}\left(z_{2 n}, z_{2 n+1}\right)\right) \leq \Omega\left(\tau_{j}\left(z_{0}, z_{1}\right)\right)-2 n v .
$$

By using (13) and (14), we have

$$
\Omega\left(\tau_{j}\left(z_{n}, z_{n+1}\right)\right) \leq \Omega\left(\tau_{j}\left(z_{0}, z_{1}\right)\right)-n v .
$$

Taking the limit as $n \longrightarrow \infty$ in both sides of (15), we get

$$
\lim _{n \rightarrow \infty} \Omega\left(\tau_{j}\left(z_{n}, z_{n+1}\right)\right)=-\infty
$$

It follows from $\Omega_{2}$ that

$$
\lim _{n \longrightarrow \infty} \tau_{j}\left(z_{n}, z_{n+1}\right)=0 .
$$

By (15), for $n \in \mathbb{N}$, we get

$$
\begin{aligned}
& \left(\tau_{j}\left(z_{n}, z_{n+1}\right)\right)^{\ell}\left[\Omega\left(\tau_{j}\left(z_{n}, z_{n+1}\right)\right)-\Omega\left(\tau_{j}\left(z_{0}, z_{1}\right)\right)\right] \\
& \quad \leq-\left(\tau_{j}\left(z_{n}, z_{n+1}\right)\right)^{\ell} n v \leq 0,
\end{aligned}
$$

for all $\ell \in \mathbb{N} \cup\{0\}$. Letting $n \longrightarrow \infty$ in (18) and applying (16) and (17), one can write

$$
\lim _{n \longrightarrow \infty} n\left(\tau_{j}\left(z_{n}, z_{n+1}\right)\right)^{\ell}=0
$$

From (19), there is $n_{1} \in \mathbb{N}$ so that $n\left(\tau_{j}\left(z_{n}, z_{n+1}\right)\right)^{\ell} \leq 1$, for all $n \geq n_{1}$. It follows that

$$
\tau_{j}\left(z_{n}, z_{n+1}\right) \leq \frac{1}{n^{1 / \ell}}, \forall n \geq n_{1} .
$$


Using (20) for $m>n>n_{1}$, we have

$$
\begin{aligned}
\tau_{j}\left(z_{n}, z_{m}\right) \leq & j\left(z_{n}, z_{m}\right)\left[\tau_{j}\left(z_{n}, z_{n+1}\right)+\tau_{j}\left(z_{n+1}, z_{m}\right)\right] \\
\leq & j\left(z_{n}, z_{m}\right) \tau_{j}\left(z_{n}, z_{n+1}\right) \\
& +j\left(z_{n}, z_{m}\right) j\left(z_{n+1}, z_{m}\right)\left[\tau_{j}\left(z_{n+1}, z_{n+2}\right)+\tau_{j}\left(z_{n+2}, z_{m}\right)\right] \\
\leq & j\left(z_{n}, z_{m}\right) \tau_{j}\left(z_{n}, z_{n+1}\right)+j\left(z_{n}, z_{m}\right) j\left(z_{n+1}, z_{m}\right) \tau_{j}\left(z_{n+1}, z_{n+2}\right) \\
& +\cdots+j\left(z_{n}, z_{m}\right) J\left(z_{n+1}, z_{m}\right) j\left(z_{n+2}, z_{m}\right) \cdots j\left(z_{m-2}, z_{m}\right) \\
& \cdot j\left(z_{m-1}, z_{m}\right) \tau_{j}\left(z_{m-1}, z_{m}\right) \\
\leq & j\left(z_{1}, z_{m}\right) j\left(z_{2}, z_{m}\right) \cdots j\left(z_{n}, z_{m}\right) \tau_{j}\left(z_{n}, z_{n+1}\right) \\
& +j\left(z_{1}, z_{m}\right) j\left(z_{2}, z_{m}\right) \cdots j\left(z_{n+1}, z_{m}\right) \tau_{j}\left(z_{n+1}, z_{n+1}\right) \\
& +\cdots+j\left(z_{1}, z_{m}\right) j\left(z_{2}, z_{m}\right) \cdots j\left(z_{m-1}, z_{m}\right) \tau_{j}\left(z_{m-1}, z_{m}\right) .
\end{aligned}
$$

It should be noted that the series

$$
\sum_{n=n_{1}}^{\infty} \tau_{j}\left(z_{n}, z_{n+1}\right) \prod_{i=1}^{n} j\left(z_{i}, z_{m}\right)
$$

converges because

$$
\begin{aligned}
\sum_{n=n_{1}}^{\infty} \tau_{j}\left(z_{n}, z_{n+1}\right) \prod_{i=1}^{n} j\left(z_{i}, z_{m}\right) & \leq \sum_{n=n_{1}}^{\infty} \frac{1}{n^{1 / \ell}} \prod_{i=1}^{n} j\left(z_{i}, z_{m}\right) \\
& \leq \frac{1}{\lambda} \sum_{n=n_{1}}^{\infty} \frac{1}{n^{1 / \ell}}
\end{aligned}
$$

which is convergent. Put $E=\sum_{n=n_{1}}^{\infty} \tau_{j}\left(z_{n}, z_{n+1}\right) \prod_{i=1}^{n} j\left(z_{i}, z_{m}\right)$ and $E_{k}=\sum_{k=1}^{\infty} \tau_{j}\left(z_{k}, z_{k+1}\right) \prod_{i=1}^{n} j\left(z_{i}, z_{m}\right)$. Thus, for $m>n>n_{1}$, inequality (20) implies that $\tau_{j}\left(z_{n}, z_{m}\right) \leq E_{m-1}-E_{n-1}$. Passing $n \longrightarrow \infty$, we conclude that $\left\{z_{n}\right\}$ is a Cauchy sequence in $\left(V, \tau_{j}\right)$ but a $¥$-space $\left(V, \tau_{j}\right)$ is complete so there exists $z \in V$ such that $\lim _{n \rightarrow \infty} z_{n}=z$.

Now, we want to show that $z$ is a common FP of $\Phi$ and $\varphi$. It is clear that

$$
\lambda \tau_{j}\left(z_{2 n+2}, \varphi z\right) \leq \lambda H\left(\Phi z_{2 n+1}, \varphi z\right) \leq \lambda H\left(\Phi z_{2 n+1}, \varphi z\right), \lambda>1 .
$$

Hence,

$$
\lambda \tau_{j}\left(z_{2 n+2}, \varphi z\right) \leq \lambda H\left(\Phi z_{2 n+1}, \varphi z\right) .
$$

Since $\Omega$ is strictly increasing, therefore

$$
\Omega\left(\lambda \tau_{j}\left(z_{2 n+2}, \varphi z\right)\right) \leq \Omega\left(\lambda H\left(\Phi z_{2 n+1}, \varphi z\right)\right) .
$$

By adding $v$ to both sides and using (4), we have

$$
\begin{aligned}
v+\Omega\left(\lambda \tau_{j}\left(z_{2 n+2}, \varphi z\right)\right) & \leq v+\Omega\left(\lambda H\left(\Phi z_{2 n+1}, \varphi z\right)\right) \\
& \leq \Omega\left(\max \left\{\tau_{j}\left(z_{2 n+1}, z\right), \frac{\left[1+\tau_{j}\left(z_{2 n+1}, \Phi z_{2 n+1}\right)\right] \tau_{j}(z, \varphi z)}{1+\tau_{j}\left(z_{2 n+1}, z\right)}, \frac{\tau_{j}\left(z_{2 n+1}, \Phi z_{2 n+1}\right) \tau_{j}(z, \varphi z)}{1+\tau_{j}\left(z_{2 n+1}, z_{2}\right)}\right\}\right) .
\end{aligned}
$$

Since $v>0$, we have

$$
\Omega\left(\lambda \tau_{j}\left(z_{2 n+2}, \varphi z\right)\right) \leq \Omega\left(\max \left\{\tau_{j}\left(z_{2 n+1}, z\right), \frac{\left[1+\tau_{j}\left(z_{2 n+1}, \Phi z_{2 n+1}\right)\right] \tau_{j}(z, \varphi z)}{1+\tau_{j}\left(z_{2 n+1}, z\right)}, \frac{\tau_{j}\left(z_{2 n+1}, \Phi z_{2 n+1}\right) \tau_{j}(z, \varphi z)}{1+\tau_{j}\left(z_{2 n+1}, z_{2}\right)}\right\}\right) .
$$

Since $\Omega$ is strictly increasing, we get

$$
\lambda \tau_{j}\left(z_{2 n+2}, \varphi z\right) \leq \max \left\{\tau_{j}\left(z_{2 n+1}, z\right), \frac{\left[1+\tau_{j}\left(z_{2 n+1}, \Phi z_{2 n+1}\right)\right] \tau_{j}(z, \varphi z)}{1+\tau_{j}\left(z_{2 n+1}, z\right)}, \frac{\tau_{j}\left(z_{2 n+1}, \Phi z_{2 n+1}\right) \tau_{j}(z, \varphi z)}{1+\tau_{j}\left(z_{2 n+1}, z_{2}\right)}\right\}
$$


Letting $n \longrightarrow \infty$ in the above inequality, one can write

$$
\lambda \tau_{j}(z, \varphi z) \leq \max \left\{0, \tau_{j}(z, \varphi z), 0\right\}=\tau_{j}(z, \varphi z) .
$$

This implies that $(\lambda-1) \tau_{j}(z, \varphi z) \leq 0$. So, we have $\tau_{j}(z$, $\varphi z) \longrightarrow 0$. Thus, $z \in \varphi z$. Similarly, one can prove that $z \in$
$\Phi z$. Therefore, $z$ is a common fixed point of $\Phi$ and $\varphi$. In order to show the uniqueness, let $z^{*}$ be another common FP of $\Phi$ and $\varphi$ so that $z^{*} \neq z$. Then, by $\Phi_{\Omega}^{r G S}$-contraction (4), one can obtain

$$
\begin{aligned}
v+\Omega\left(\lambda \tau_{j}\left(z, z^{*}\right)\right) & \leq v+\Omega\left(\lambda H\left(\Phi z, \varphi z^{*}\right)\right) \\
& \leq \Omega\left(\max \left\{\tau_{j}\left(z, z^{*}\right), \frac{\left[1+\tau_{j}(z, \Phi z)\right] \tau_{j}\left(z^{*}, \varphi z^{*}\right)}{1+\tau_{j}\left(z, z^{*}\right)}, \frac{\tau_{j}(z, \Phi z) \tau_{j}\left(+\tau_{j}\left(z, z^{*}\right), \varphi+\tau_{j}\left(z, z^{*}\right)\right)}{+\tau_{j}\left(z, z^{*}\right)}\right\}\right)=\Omega\left(\tau_{j}\left(z, z^{*}\right)\right) .
\end{aligned}
$$

Based on the properties of $\Omega$ and since $v>0$, we conclude that $(1-\lambda) \tau_{j}\left(z, z^{*}\right) \leq 0$. This means $\tau_{j}\left(z, z^{*}\right) \longrightarrow 0$, i.e., $z=z^{*}$. This finishes the proof.

Remark 9. The contraction condition of Theorem 8 is reduced to the following: (i) $\Phi_{\varphi, \Omega}^{r G S}$-contraction in $b$-metric-like space, if $j(y, z)=$
$j(\geq 1)$

(ii) $\Phi_{\varphi, \Omega}^{r G S}$-contraction in metric-like space, if $j(y, z)=1$

(iii) $\Phi_{\Omega}^{r G S}$-contraction in ¥-spaces, if $\Phi=\varphi$

(iv) $\Phi_{\Omega}^{r G S}$-contraction in $b$-metric-like space, if $\Phi=\varphi$ and $j(y, z)=j(\geq 1)$

(v) $\Phi_{\Omega}^{r G S}$-contraction in $b$-metric-like space, if $j(y, z)=1$ and $\Phi=\varphi$

Corollary 10. Theorem 8 is still valid if one of the following forms is chosen for $\beta\left(z_{1}, z_{2}\right)$ in Equation (5):

(1) $\beta\left(z_{1}, z_{2}\right)=\tau_{j}\left(z_{1}, z_{2}\right)$

(2) $\beta\left(z_{1}, z_{2}\right)=\left[1+\tau_{j}\left(z_{1}, \Phi z_{1}\right)\right] \tau_{j}\left(z_{2}, \varphi z_{2}\right) / 1+\tau_{j}\left(z_{1}, z_{2}\right)$

(3) $\beta\left(z_{1}, z_{2}\right)=\left[1+\tau_{j}\left(z_{1}, \Phi z_{1}\right)\right] \tau_{j}\left(z_{2}, \varphi z_{2}\right) / 1+\tau_{j}\left(z_{1}, z_{2}\right)$

(4) $\beta\left(z_{1}, z_{2}\right)=\max \left\{\tau_{j}\left(z_{1}, z_{2}\right),\left[1+\tau_{j}\left(z_{1}, \Phi z_{1}\right)\right] \tau_{j}\left(z_{2}\right.\right.$, $\left.\left.\varphi z_{2}\right) / 1+\tau_{j}\left(z_{1}, z_{2}\right)\right\}$

(5) $\beta\left(z_{1}, z_{2}\right)=\max \left\{\tau_{j}\left(z_{1}, z_{2}\right), \tau_{j}\left(z_{1}, \Phi z_{1}\right) \tau_{j}\left(z_{2}, \varphi z_{2}\right) / \tau_{j}\right.$ $\left.\left(z_{1}, \Phi z_{1}\right) \tau_{j}\left(z_{2}, \varphi z_{2}\right)\right\}$

(6) $\beta\left(z_{1}, z_{2}\right)=\max \left\{\left[1+\tau_{j}\left(z_{1}, \Phi z_{1}\right)\right] \tau_{j}\left(z_{2}, \varphi z_{2}\right) / \tau_{j}\left(z_{1}\right.\right.$, $\left.\left.\Phi z_{1}\right) \tau_{j}\left(z_{2}, \varphi z_{2}\right), \tau_{j}\left(z_{1}, \Phi z_{1}\right) \tau_{j}\left(z_{2}, \varphi z_{2}\right) / 1+\tau_{j}\left(z_{1}, z_{2}\right)\right\}$

\section{Illustrative Examples}

In this section, we give some illustrative examples to support Theorem 8 and its consequences.
Example 1. Let $V=[0,1]$. Define $\tau_{j}: V \times V \longrightarrow \mathbb{R}^{+}$by $\tau_{j}\left(z_{1}\right.$, $\left.z_{2}\right)=\left(z_{1}+z_{2}\right)^{2}$ for all $z_{1}, z_{2} \in V$; then, the pair $\left(V, \tau_{j}\right)$ is $¥$-space with $j\left(z_{1}, z_{2}\right)=2+\left(z_{1}\right)^{2}+\left(z_{2}\right)^{2}$. Define $\Phi, \varphi: V$ $\longrightarrow V_{\mathrm{CB}}$ and $\Omega: \mathbb{R}^{+} \longrightarrow \mathbb{R}^{+}$by

$$
\Phi z_{1}=\left[0, \frac{z_{1}}{3}\right], \varphi z_{2}=\left[0, \frac{z_{2}}{3}\right] \text {, for all } z_{1}, z_{2} \in[0,1], \lambda>1,
$$

and $\Omega(\sigma)=\ln (\sigma)$, for all $\sigma>0$, respectively. Now, we have

$$
\begin{aligned}
3 H\left(\Phi z_{1}, \varphi z_{2}\right) & =3 H\left(\left[0, \frac{z_{1}}{3}\right],\left[0, \frac{z_{2}}{3}\right]\right) \leq 3\left(\frac{z_{1}}{3}+\frac{z_{2}}{3}\right)^{2} \\
& =\frac{1}{3}\left(z_{1}+z_{2}\right)^{2}=\frac{1}{3} \tau_{j}\left(z_{1}, z_{2}\right) \leq \frac{1}{3} \beta\left(z_{1}, z_{2}\right) .
\end{aligned}
$$

Taking $\log$ to the base $e$ on both sides, we get

$$
\begin{aligned}
\ln \left(3 H\left(\Phi z_{1}, \varphi z_{2}\right)\right) & \leq \ln \left(\frac{1}{3} \beta\left(z_{1}, z_{2}\right)\right) \\
& =\ln \left(\frac{1}{3}\right)+\ln \left(\beta\left(z_{1}, z_{2}\right) .\right.
\end{aligned}
$$

This implies that

$$
\begin{aligned}
& \ln \left(3 H\left(\Phi z_{1}, \varphi z_{2}\right)\right)-\ln \left(\beta\left(z_{1}, z_{2}\right)\right. \\
& \quad \leq-\ln (3) \Rightarrow \Omega\left(\lambda H\left(\Phi z_{1}, \varphi z_{2}\right)\right)-\Omega\left(\beta\left(z_{1}, z_{2}\right) \leq-v .\right.
\end{aligned}
$$

Hence, the contraction condition (7) is fulfilled with $\lambda=3>1$ and $v=\ln (3)>0$. Thus, all stipulations of Theorem 8 are fulfilled, and 0 is a unique common FP of $\Phi$ and $\varphi$.

Example 2. Assume that $V=[0,1]$. Define a distance function $\tau_{j}$ over a set $V$ by $\tau_{j}\left(z_{1}, z_{2}\right)=\left|z_{1}+z_{2}\right|^{2}$ and $j: V \times V \longrightarrow$ $[1, \infty)$ by $j\left(z_{1}, z_{2}\right)=2+2 z_{1}+3 z_{2}$. Then, $\left(V, \tau_{j}\right)$ forms a $¥-$ 
space. Define multivalued mappings $\Phi, \varphi: V \longrightarrow V_{C B}$ by $\Phi(z)=2 / 15\{z\}$, for all $z \in[0,1]$ and

$$
\varphi z= \begin{cases}\frac{2}{10}\{z\}, & \text { if } z \in[0,1) \\ 0, & \text { if } z=1 .\end{cases}
$$

Define the function $\Omega: \mathbb{R}^{+} \longrightarrow \mathbb{R}^{+}$by $\Omega(\sigma)=\ln \left(\sigma^{2}+\right.$ $\sigma)$ for all $\sigma>0, v>0$, and $\lambda=5 / 2$.

Now, to fulfill the conditions of Theorem 8 , we discuss the following cases:
Case 1. If $z_{1} \in[0,1)$ and $z_{2}=1$, consider

$$
\begin{aligned}
\Omega\left(\lambda H\left(\Phi z_{1}, \varphi z_{2}\right)\right. & =\Omega\left(\frac{5}{2} H\left(\frac{2}{15}\left\{z_{1}\right\}, 0\right)\right) \\
& =\Omega\left(\frac{1}{3} H\left(\left\{z_{1}\right\}, 0\right)\right) \leq \Omega\left(\frac{1}{3}\left(z_{1}+0\right)^{2}\right) \\
& =\ln \left(\frac{\left(z_{1}\right)^{4}}{9}+\frac{\left(z_{1}\right)^{2}}{3}\right) .
\end{aligned}
$$

Now, consider

$$
\begin{aligned}
\beta\left(z_{1}, z_{2}\right) & =\max \left\{\tau_{j}\left(z_{1}, 1\right), \frac{\left[1+\tau_{j}\left(z_{1}, 2 / 15\left\{z_{1}\right\}\right)\right] \tau_{j}(1,0)}{1+\tau_{j}\left(z_{1}, 1\right)}, \frac{\tau_{j}\left(z_{1}, 2 / 15\left\{z_{1}\right\}\right) \tau_{j}(1,0)}{1+\tau_{j}\left(z_{1}, 1\right)}\right\} \\
& =\max \left\{\left(z_{1}+1\right)^{2}, \frac{1+514 / 225\left(z_{1}\right)^{2}}{1+\left(z_{1}+1\right)^{2}}, \frac{289 / 225\left(z_{1}\right)^{2}}{1+\left(z_{1}+1\right)^{2}}\right\}=\left(z_{1}+1\right)^{2} .
\end{aligned}
$$

Therefore,

$$
\Omega\left(\beta\left(z_{1}, z_{2}\right)\right)=\Omega\left(\left(z_{1}+1\right)^{2}\right)=\ln \left(\left(z_{1}+1\right)^{4}+\left(z_{1}+1\right)^{2}\right) .
$$

Thus,

$$
\begin{aligned}
\Omega\left(\lambda H\left(\Phi z_{1}, \varphi z_{2}\right)-\Omega\left(\beta\left(z_{1}, z_{2}\right)\right)\right. & \leq \ln \left(\frac{\left(z_{1}\right)^{4} / 9+\left(z_{1}\right)^{2} / 3}{\left(z_{1}+1\right)^{4}+\left(z_{1}+1\right)^{2}}\right) \\
& =-v,
\end{aligned}
$$

Case 2. If $z_{2} \in[0,1)$ and $z_{1}=1$, then $\Phi\left(z_{1}\right)=\Phi(1)=2 / 15$ and $\varphi z_{2}=1 / 5\left\{z_{2}\right\}$.

\section{Consider}

$$
\begin{aligned}
\Omega\left(\lambda H\left(\Phi z_{1}, \varphi z_{2}\right)\right. & =\Omega\left(\frac{5}{2} H\left(\frac{2}{15}, \frac{2}{10}\left\{z_{2}\right\}\right)\right) \\
& =\Omega\left(H\left(\frac{1}{3}, \frac{1}{2}\left\{z_{2}\right\}\right)\right) \leq \Omega\left(\left(\frac{1}{3}+z_{2}\right)^{2}\right) \\
& =\ln \left(\left(\frac{1}{3}+z_{2}\right)^{4}+\left(\frac{1}{3}+z_{2}\right)^{2}\right) .
\end{aligned}
$$

for any value of $0 \leq z_{1}<1$ and $v>0$. Therefore,

$$
v+\Omega\left(\lambda H\left(\Phi z_{1}, \varphi z_{2}\right) \leq \Omega\left(\beta\left(z_{1}, z_{2}\right)\right)\right.
$$

Now, consider

$$
\begin{aligned}
\beta\left(z_{1}, z_{2}\right) & =\max \left\{\tau_{j}\left(1, z_{2}\right), \frac{\left[1+\tau_{j}(1,2 / 15)\right] \tau_{j}\left(z_{2}, 1 / 5\left\{z_{2}\right\}\right)}{1+\tau_{j}\left(1, z_{2}\right)}, \frac{\tau_{j}(1,2 / 15) \tau_{j}\left(z_{2}, 1 / 5\left\{z_{2}\right\}\right)}{1+\tau_{j}\left(1, z_{2}\right)}\right\} \\
& =\max \left\{\left(1+z_{2}\right)^{2}, \frac{514 / 225.36 / 25\left(z_{2}\right)^{2}}{1+\left(1+z_{2}\right)^{2}}, \frac{289 / 225.36 / 25\left(z_{2}\right)^{2}}{1+\left(1+z_{2}\right)^{2}}\right\}=\left(1+z_{2}\right)^{2} .
\end{aligned}
$$

Therefore,

$$
\Omega\left(\beta\left(z_{1}, z_{2}\right)\right)=\Omega\left(\left(1+z_{2}\right)^{2}\right)=\ln \left(\left(1+z_{2}\right)^{4}+\left(1+z_{2}\right)^{2}\right) .
$$

Thus,

$$
\begin{aligned}
& \Omega( \lambda H\left(\Phi z_{1}, \varphi z_{2}\right)-\Omega\left(\beta\left(z_{1}, z_{2}\right)\right) \\
& \quad \leq \ln \left(\frac{\left(1 / 3+z_{2}\right)^{4}+\left(1 / 3+z_{2}\right)^{2}}{\left(1+z_{2}\right)^{4}+\left(1+z_{2}\right)^{2}}\right)=-v,
\end{aligned}
$$


for any value of $0 \leq z_{2}<1$ and $v>0$. Therefore,

$$
v+\Omega\left(\lambda H\left(\Phi z_{1}, \varphi z_{2}\right) \leq \Omega\left(\beta\left(z_{1}, z_{2}\right)\right) .\right.
$$

Thus, all requirements of Theorem 8 are fulfilled, and 0 is a unique common FP of $\Phi$ and $\varphi$.

Example 3. Assume that $V=[0,1]$. Define a distance function $\tau_{j}$ over a set $V$ by $\tau_{j}\left(z_{1}, z_{2}\right)=\left|z_{1}\right|+\left|z_{2}\right|+\left|z_{1}\right|^{2} / 4+\left|z_{2}\right|^{2} / 5$ and $j: V \times V \longrightarrow[1, \infty)$ by $j\left(z_{1}, z_{2}\right)=\left|z_{1}+z_{2}\right|^{2}$. Then, $\left(V, \tau_{j}\right)$ forms a $¥$-space. Define multivalued mappings $\Phi, \varphi$ : $V \longrightarrow V_{C B}$ by

$$
\Phi z=\varphi z= \begin{cases}\left\{\frac{z^{2}}{2}\right\}, & \text { if } z \in\left[0, \frac{1}{2}\right), \\ \left\{\frac{\log (3 z)}{4}\right\}, & \text { if } z \in\left[\frac{1}{2}, 1\right] .\end{cases}
$$

Define the function $\Omega: \mathbb{R}^{+} \longrightarrow \mathbb{R}^{+}$by $\Omega(\sigma)=\log (\sigma)$ for all $\sigma>0, v>0$, and $\lambda=2>1$.

Case 1. If $z_{1}, z_{2} \in[0,1 / 2)$, then $\varphi z_{1}=\left\{z_{1}^{2} / 2\right\}$ and $\varphi z_{2}=\left\{z_{2}^{2} / 2\right\}$. Consider

$$
\begin{aligned}
\Omega\left(\lambda H\left(\varphi z_{1}, \varphi z_{2}\right)\right. & =\Omega\left(2 H\left(\left\{\frac{z_{1}^{2}}{2}\right\},\left\{\frac{z_{2}^{2}}{2}\right\}\right)\right) \\
& \leq \Omega\left(\left|z_{1}\right|^{2}+\left|z_{2}\right|^{2}+\frac{\left|z_{1}\right|^{4}}{4}+\frac{\left|z_{2}\right|^{4}}{5}\right) \\
& =\log \left(\left|z_{1}\right|^{2}+\left|z_{2}\right|^{2}+\frac{\left|z_{1}\right|^{4}}{4}+\frac{\left|z_{2}\right|^{4}}{5}\right) .
\end{aligned}
$$

Now, consider

$$
\begin{aligned}
\beta\left(z_{1}, z_{2}\right)= & \max \left\{\begin{array}{c}
\left.\tau_{j}\left(z_{1}, z_{2}\right), \frac{\left[1+\tau_{j}\left(z_{1}, z_{1}^{2} / 2\right)\right] \tau_{j}\left(z_{2}, z_{2}^{2} / 2\right)}{1+\tau_{j}\left(z_{1}, z_{2}\right)}, \frac{\tau_{j}\left(z_{1}, z_{1}^{2} / 2\right) \tau_{j}\left(z_{2}, z_{2}^{2} / 2\right)}{1+\tau_{j}\left(z_{1}, z_{2}\right)}\right\} \\
\left|z_{1}\right|+\left|z_{2}\right|+\frac{\left|z_{1}\right|^{2}}{4}+\frac{\left|z_{2}\right|^{2}}{5}, \\
=\max \left\{\begin{array}{c}
\frac{\left(1+\left|z_{1}\right|+\left|z_{1}\right| / 2+\left|z_{1}\right|^{2} / 4+\left|z_{1}\right|^{4} / 20\right)\left(\left|z_{2}\right|+\left|z_{2}\right| / 2+\left|z_{2}\right|^{2} / 4+\left|z_{2}\right|^{4} / 20\right)}{1+\left|z_{1}\right|+\left|z_{2}\right|+\left|z_{1}\right|^{2} / 4+\left|z_{2}\right|^{2} / 5}, \\
\frac{\left(\left|z_{1}\right|+\left|z_{1}\right| / 2+\left|z_{1}\right|^{2} / 4+\left|z_{1}\right|^{4} / 20\right)\left(\left|z_{2}\right|+\left|z_{2}\right| / 2+\left|z_{2}\right|^{2} / 4+\left|z_{2}\right|^{4} / 20\right)}{1+\left|z_{1}\right|+\left|z_{2}\right|+\left|z_{1}\right|^{2} / 4+\left|z_{2}\right|^{2} / 5}
\end{array}\right\} \\
=\left|z_{1}\right|+\left|z_{2}\right|+\frac{\left|z_{1}\right|^{2}}{4}+\frac{\left|z_{2}\right|^{2}}{5} .
\end{array}\right.
\end{aligned}
$$

Therefore,

$$
\begin{aligned}
\Omega\left(\beta\left(z_{1}, z_{2}\right)\right) & =\Omega\left(\left|z_{1}\right|+\left|z_{2}\right|+\frac{\left|z_{1}\right|^{2}}{4}+\frac{\left|z_{2}\right|^{2}}{5}\right) \\
& =\log \left(\left|z_{1}\right|+\left|z_{2}\right|+\frac{\left|z_{1}\right|^{2}}{4}+\frac{\left|z_{2}\right|^{2}}{5}\right) .
\end{aligned}
$$

Thus,

$$
\begin{aligned}
\Omega & \lambda H\left(\varphi z_{1}, \varphi z_{2}\right)-\Omega\left(\beta\left(z_{1}, z_{2}\right)\right) \\
& \leq \log \left(\frac{\left|z_{1}\right|^{2}+\left|z_{2}\right|^{2}+\left|z_{1}\right|^{4} / 4+\left|z_{2}\right|^{4} / 5}{\left|z_{1}\right|+\left|z_{2}\right|+\left|z_{1}\right|^{2} / 4+\left|z_{2}\right|^{2} / 5}\right) \\
& =-v, \text { for some } v>0 .
\end{aligned}
$$

Hence, $\quad \Omega\left(\lambda H\left(\varphi z_{1}, \varphi z_{2}\right)-\Omega\left(\beta\left(z_{1}, z_{2}\right)\right) \leq-v, \quad\right.$ which implies

$$
v+\Omega\left(\lambda H\left(\varphi z_{1}, \varphi z_{2}\right) \leq \Omega\left(\beta\left(z_{1}, z_{2}\right)\right) .\right.
$$

Case 2. If $\left.z_{1}, z_{2} \in 1 / 2,1\right]$, then $\varphi z_{1}=\left\{\log \left(3 z_{1}\right) / 4\right\}$ and $\varphi z_{2}=$ $\left\{\log \left(3 z_{2}\right) / 4\right\}$. Consider

$$
\begin{aligned}
\Omega & \lambda H\left(\varphi z_{1}, \varphi z_{2}\right) \\
& =\Omega\left(2 H\left(\left\{\frac{\log \left(3 z_{1}\right)}{4}\right\},\left\{\frac{\log \left(3 z_{2}\right)}{4}\right\}\right)\right) \\
& =\Omega\left(H\left(\left\{\frac{\log \left(3 z_{1}\right)}{2}\right\},\left\{\frac{\log \left(3 z_{2}\right)}{2}\right\}\right)\right) \\
& \leq \Omega\left(\left|\frac{\log \left(3 z_{1}\right)}{2}\right|+\left|\frac{\log \left(3 z_{2}\right)}{2}\right|+\frac{\left|\log \left(3 z_{1}\right)\right|^{2}}{16}+\frac{\left|\log \left(3 z_{2}\right)\right|^{2}}{20}\right) \\
& =\log \left(\left|\frac{\log \left(3 z_{1}\right)}{2}\right|+\left|\frac{\log \left(3 z_{2}\right)}{2}\right|+\frac{\left|\log \left(3 z_{1}\right)\right|^{2}}{16}+\frac{\left|\log \left(3 z_{2}\right)\right|^{2}}{20}\right) .
\end{aligned}
$$


Now, consider

$$
\begin{aligned}
\beta\left(z_{1}, z_{2}\right)=\max \left\{\tau_{j}\left(z_{1}, z_{2}\right), \frac{\left[1+\tau_{j}\left(z_{1}, \log \left(3 z_{1}\right) / 4\right)\right] \tau_{j}\left(z_{2}, \log \left(3 z_{2}\right) / 4\right)}{1+\tau_{j}\left(z_{1}, z_{2}\right)}, \frac{\tau_{j}\left(z_{1}, \log \left(3 z_{1}\right) / 4\right) \tau_{j}\left(z_{2}, \log \left(3 z_{2}\right) / 4\right)}{1+\tau_{j}\left(z_{1}, z_{2}\right)}\right\} \\
=\max \left\{\begin{array}{l}
\frac{\left(1+\left|z_{1}\right|+\left|z_{2}\right|+\frac{\left|z_{1}\right|^{2}}{4}+\frac{\left|z_{2}\right|^{2}}{5},\right.}{\left.1+\left|\log \left(3 z_{1}\right) / 4\right|+\left|z_{1}\right|^{2} / 4+\left|\log \left(3 z_{1}\right)\right|^{2} / 80\right)\left(\left|z_{2}\right|+\left|\log \left(3 z_{2}\right) / 4\right|+\left|z_{2}\right|^{2} / 4+\left|\log \left(3 z_{2}\right)\right|^{2} / 80\right)} \\
\frac{\left(\left|z_{1}\right|+\left|\log \left(3 z_{1}\right) / 4\right|+\left|z_{1}\right|^{2} / 4+\left|\log \left(3 z_{1}\right)\right|^{2} / 80\right)\left(\left|z_{1}\right|+\left|\log \left(3 z_{2}\right) / 4\right|+\left|z_{2}\right|^{2} / 4+\left|\log \left(3 z_{2}\right)\right|^{2} / 80\right)}{1+\left|z_{1}\right|+\left|z_{2}\right|+\left|z_{1}\right|^{2} / 4+\left|z_{2}\right|^{2} / 5}
\end{array}\right\} \\
=\left|z_{1}\right|+\left|z_{2}\right|+\frac{\left|z_{1}\right|^{2}}{4}+\frac{\left|z_{2}\right|^{2}}{5} .
\end{aligned}
$$

Therefore,

$$
\begin{aligned}
\Omega\left(\beta\left(z_{1}, z_{2}\right)\right) & =\Omega\left(\left|z_{1}\right|+\left|z_{2}\right|+\frac{\left|z_{1}\right|^{2}}{4}+\frac{\left|z_{2}\right|^{2}}{5}\right) \\
& =\log \left(\left|z_{1}\right|+\left|z_{2}\right|+\frac{\left|z_{1}\right|^{2}}{4}+\frac{\left|z_{2}\right|^{2}}{5}\right) .
\end{aligned}
$$

Thus,

$$
\begin{aligned}
\Omega\left(\lambda H\left(\varphi z_{1}, \varphi z_{2}\right)-\Omega\left(\beta\left(z_{1}, z_{2}\right)\right)\right. & \leq \log \left(\frac{\left|\log \left(3 z_{1}\right) / 2\right|+\left|\log \left(3 z_{2}\right) / 2\right|+\left|\log \left(3 z_{1}\right)\right|^{2} / 16+\left|\log \left(3 z_{2}\right)\right|^{2} / 20}{\left|z_{1}\right|+\left|z_{2}\right|+\left|z_{1}\right|^{2} / 4+\left|z_{2}\right|^{2} / 5}\right) \\
& =-v, \text { for some } v>0 .
\end{aligned}
$$

Hence, $\Omega\left(\lambda H\left(\varphi z_{1}, \varphi z_{2}\right)-\Omega\left(\beta\left(z_{1}, z_{2}\right)\right) \leq-v, \quad\right.$ which implies

$$
v+\Omega\left(\lambda H\left(\varphi z_{1}, \varphi z_{2}\right) \leq \Omega\left(\beta\left(z_{1}, z_{2}\right)\right)\right.
$$

Case 3. If $z_{1} \in[0,1 / 2)$ and $z_{2} \in[1 / 2,1]$, then $\varphi z_{1}=\left\{z_{1}^{2} / 2\right\}$ and $\varphi z_{2}=\left\{\log \left(3 z_{2}\right) / 4\right\}$. Consider

$$
\Omega\left(\lambda H\left(\varphi z_{1}, \varphi z_{2}\right)=\Omega\left(2 H\left(\left\{\frac{z_{1}^{2}}{2}\right\},\left\{\frac{\log \left(3 z_{2}\right)}{4}\right\}\right)\right)\right.
$$

$$
\begin{aligned}
& =\Omega\left(H\left(\left\{z_{1}^{2}\right\},\left\{\frac{\log \left(3 z_{2}\right)}{2}\right\}\right)\right) \\
& \leq \Omega\left(\left|z_{1}\right|^{2}+\left|\frac{\log \left(3 z_{2}\right)}{2}\right|+\frac{\left|z_{1}\right|^{4}}{4}+\frac{\left|\log \left(3 z_{2}\right)\right|^{2}}{20}\right) \\
& =\log \left(\left|z_{1}\right|^{2}+\left|\frac{\log \left(3 z_{2}\right)}{2}\right|+\frac{\left|z_{1}\right|^{4}}{4}+\frac{\left|\log \left(3 z_{2}\right)\right|^{2}}{20}\right) .
\end{aligned}
$$

Now, consider

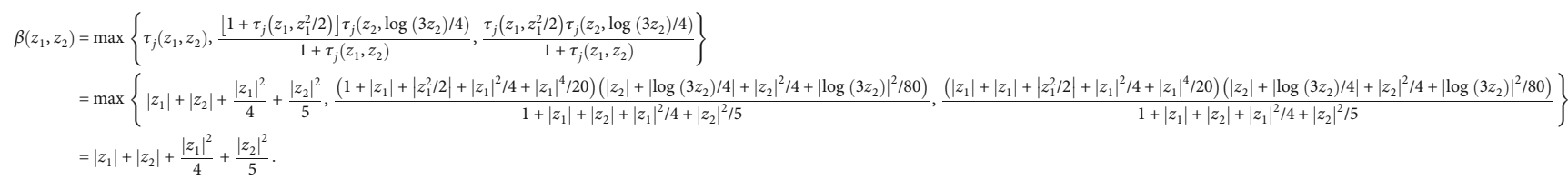


Therefore,

$$
\begin{aligned}
\Omega\left(\beta\left(z_{1}, z_{2}\right)\right) & =\Omega\left(\left|z_{1}\right|+\left|z_{2}\right|+\frac{\left|z_{1}\right|^{2}}{4}+\frac{\left|z_{2}\right|^{2}}{5}\right) \\
& =\log \left(\left|z_{1}\right|+\left|z_{2}\right|+\frac{\left|z_{1}\right|^{2}}{4}+\frac{\left|z_{2}\right|^{2}}{5}\right) .
\end{aligned}
$$

Thus,

$$
\begin{aligned}
\Omega & \lambda H\left(\varphi z_{1}, \varphi z_{2}\right)-\Omega\left(\beta\left(z_{1}, z_{2}\right)\right) \\
& \leq \log \left(\frac{\left|z_{1}\right|^{2}+\left|\log \left(3 z_{2}\right) / 2\right|+\left|z_{1}\right|^{4} / 4+\left|\log \left(3 z_{2}\right)\right|^{2} / 20}{\left|z_{1}\right|+\left|z_{2}\right|+\left|z_{1}\right|^{2} / 4+\left|z_{2}\right|^{2} / 5}\right) \\
& =-v, \text { for some } v>0 .
\end{aligned}
$$

Hence, $\quad \Omega\left(\lambda H\left(\varphi z_{1}, \varphi z_{2}\right)-\Omega\left(\beta\left(z_{1}, z_{2}\right)\right) \leq-v, \quad\right.$ which implies

$$
v+\Omega\left(\lambda H\left(\varphi z_{1}, \varphi z_{2}\right) \leq \Omega\left(\beta\left(z_{1}, z_{2}\right)\right) .\right.
$$

Case 4. If $z_{1} \in[1 / 2,1]$ and $z_{2} \in[0,1 / 2)$, then $\varphi z_{1}=\left\{\log \left(3 z_{1}\right) /\right.$ $4\}$ and $\varphi z_{2}=\left\{z_{2}^{2} / 2\right\}$. Consider

$$
\begin{aligned}
\Omega\left(\lambda H\left(\varphi z_{1}, \varphi z_{2}\right)\right. & =\Omega\left(2 H\left(\left\{\frac{\log \left(3 z_{1}\right)}{4}\right\},\left\{\frac{z_{2}^{2}}{2}\right\}\right)\right) \\
& =\Omega\left(H\left(\left\{\frac{\log \left(3 z_{1}\right)}{2}\right\},\left\{z_{2}^{2}\right\}\right)\right) \\
& \leq \Omega\left(\left|\frac{\log \left(3 z_{1}\right)}{2}\right|+\left|z_{2}\right|^{2}+\frac{\left|\log \left(3 z_{1}\right)\right|^{2}}{20}+\frac{\left|z_{2}\right|^{4}}{4}\right) \\
& =\log \left(\left|\frac{\log \left(3 z_{1}\right)}{2}\right|+\left|z_{2}\right|^{2}+\frac{\left|\log \left(3 z_{1}\right)\right|^{2}}{20}+\frac{\left|z_{2}\right|^{4}}{4}\right) .
\end{aligned}
$$

Now, consider

$$
\begin{aligned}
& \beta\left(z_{1}, z_{2}\right)=\max \left\{\tau_{j}\left(z_{1}, z_{2}\right), \frac{\left[1+\tau_{j}\left(z_{1}, \log \left(3 z_{1}\right) / 4\right)\right] \tau_{j}\left(z_{2}, z_{2}^{2} / 2\right)}{1+\tau_{j}\left(z_{1}, z_{2}\right)}, \frac{\tau_{j}\left(z_{1}, \log \left(3 z_{1}\right) / 4\right) \tau_{j}\left(z_{2}, z_{2}^{2} / 2\right)}{1+\tau_{j}\left(z_{1}, z_{2}\right)}\right\} \\
& =\max \left\{\left|z_{1}\right|+\left|z_{2}\right|+\frac{\left|z_{1}\right|^{2}}{4}+\frac{\left|z_{2}\right|^{2}}{5}, \frac{\left(1+\left|z_{1}\right|+\left|\log \left(3 z_{1}\right) / 4\right|+\left|z_{1}\right|^{2} / 4+\left|\log \left(3 z_{1}\right)\right|^{2} / 80\right)\left(\left|z_{z}\right|+\left|z_{2}^{2} / 2\right|+\left|z_{2}\right|^{2} / 4+\left|z_{2}\right|^{4} / 20\right)}{1+\left|z_{1}\right|+\left|z_{2}\right|+\left|z_{1}\right|^{2} / 4+\left|z_{2}\right|^{2} / 5}, \frac{\left(1+\left|z_{1}\right|+\left|\log \left(3 z_{1}\right) / 4\right|+\left|z_{1}\right|^{2} / 4+\mid \log \left(3 z_{1}\right)^{2} / 80\right)\left(\left|z_{2}\right|+\left|z_{2}^{2} / 2\right|+\left|z_{2}\right|^{2} / 4+\left|z_{2}\right|^{4} / 20\right)}{1+\left|z_{1}\right|+\left|z_{2}\right|+\left|z_{1}\right|^{2} / 4+\left|z_{2}\right|^{2} \mid / 4}\right\} \\
& =\left|z_{1}\right|+\left|z_{2}\right|+\frac{\left|z_{1}\right|^{2}}{4}+\frac{\left|z_{2}\right|^{2}}{5} \text {. }
\end{aligned}
$$

Therefore,

$$
\begin{aligned}
\Omega\left(\beta\left(z_{1}, z_{2}\right)\right) & =\Omega\left(\left|z_{1}\right|+\left|z_{2}\right|+\frac{\left|z_{1}\right|^{2}}{4}+\frac{\left|z_{2}\right|^{2}}{5}\right) \\
& =\log \left(\left|z_{1}\right|+\left|z_{2}\right|+\frac{\left|z_{1}\right|^{2}}{4}+\frac{\left|z_{2}\right|^{2}}{5}\right) .
\end{aligned}
$$

Thus,

$$
\begin{aligned}
\Omega & \left(\lambda H\left(\varphi z_{1}, \varphi z_{2}\right)-\Omega\left(\beta\left(z_{1}, z_{2}\right)\right)\right. \\
& \leq \log \left(\frac{\left|\log \left(3 z_{1}\right) / 2\right|+\left|z_{2}\right|^{2}+\left|\log \left(3 z_{1}\right)\right|^{2} / 20+\left|z_{2}\right|^{4} / 4}{\left|z_{1}\right|+\left|z_{2}\right|+\left|z_{1}\right|^{2} / 4+\left|z_{2}\right|^{2} / 5}\right) \\
& =-v, \text { for some } v>0 .
\end{aligned}
$$

Hence, $\quad \Omega\left(\lambda H\left(\varphi z_{1}, \varphi z_{2}\right)-\Omega\left(\beta\left(z_{1}, z_{2}\right)\right) \leq-v, \quad\right.$ which implies

$$
v+\Omega\left(\lambda H\left(\varphi z_{1}, \varphi z_{2}\right) \leq \Omega\left(\beta\left(z_{1}, z_{2}\right)\right) .\right.
$$

From the four cases, we conclude the contraction condition (iii) of Remark 9 is fulfilled and 0 is a unique FP of $\varphi$.

Example 4. Let $V=\left\{\left\{\mathfrak{I}_{t}\right\}: t \in\{1,2, \cdots, 100\}\right\}$ be a sequence defined by

$$
\mathfrak{I}_{1}=1.2, \mathfrak{\Im}_{2}=2.3, \cdots, \mathfrak{\Im}_{t}=t(t+1) .
$$

Suppose that $\tau_{j}: V \times V \longrightarrow(0, \infty)$ and $j: V \times V \longrightarrow[1$, $\infty)$ are functions described by $\tau_{j}\left(z_{1}, z_{2}\right)=\left(\max \left\{z_{1}, z_{2}\right\}\right)^{2}$ and $j\left(z_{1}, z_{2}\right)=1+z_{1}+z_{2}$, respectively, for all $z_{1}, z_{2} \in V$. Then, $\left(V, \tau_{j}\right)$ is $¥-$ space. Define the multivalued mappings $\Phi, \varphi: V \longrightarrow V_{\mathrm{CB}}$ and $\Omega: \mathbb{R}^{+} \longrightarrow \mathbb{R}^{+}$by

$$
\Phi \mathfrak{I}_{1}=\varphi \mathfrak{I}_{1}=\left\{\mathfrak{I}_{1}\right\}, \Phi \mathfrak{I}_{t}=\varphi \mathfrak{I}_{t}=\left\{\mathfrak{I}_{t-1}\right\}
$$

and $\Omega(\sigma)=\ln (\sigma)$, for all $\sigma>0$, respectively. Now, we have the following calculations for two cases: 
Case 1. For each $\in \mathbb{N}, s>2$, and $t=1$, we have

$$
\begin{aligned}
& \frac{H\left(\varphi \mathfrak{I}_{s}, \varphi \mathfrak{I}_{1}\right)}{\beta\left(\mathfrak{I}_{s}, \mathfrak{J}_{1}\right)}=\frac{\left(\max \left\{\mathfrak{I}_{s-1}, \mathfrak{J}_{1}\right\}\right)^{2}}{\max \left\{\tau_{j}\left(\mathfrak{J}_{s}, \mathfrak{J}_{1}\right),\left[1+\tau_{j}\left(\mathfrak{J}_{s}, \varphi \mathfrak{I}_{s}\right)\right] \tau_{j}\left(\mathfrak{J}_{1}, \varphi \mathfrak{I}_{1}\right) / 1+\tau_{j}\left(\mathfrak{J}_{s}, \mathfrak{J}_{1}\right), \tau_{j}\left(\mathfrak{J}_{s}, \varphi \mathfrak{J}_{s}\right) \tau_{j}\left(\mathfrak{I}_{1}, \varphi \mathfrak{J}_{1}\right) / 1+\tau_{j}\left(\mathfrak{J}_{s}, \mathfrak{J}_{1}\right)\right\}} \\
& =\frac{\left(\max \left\{\mathfrak{I}_{s-1}, \mathfrak{I}_{1}\right\}\right)^{2}}{\max \left\{\tau_{j}\left(\mathfrak{I}_{s}, \mathfrak{J}_{1}\right),\left[1+\tau_{j}\left(\mathfrak{J}_{s}, \mathfrak{I}_{s-1}\right)\right] \tau_{j}\left(\mathfrak{J}_{1}, \mathfrak{I}_{1}\right) / 1+\tau_{j}\left(\mathfrak{I}_{s}, \mathfrak{I}_{t}\right), \tau_{j}\left(\mathfrak{I}_{s}, \mathfrak{J}_{s-1}\right) \tau_{j}\left(\mathfrak{I}_{1}, \mathfrak{I}_{1}\right) / 1+\tau_{j}\left(\mathfrak{I}_{s}, \mathfrak{J}_{t}\right)\right\}} \\
& =\frac{\mathfrak{I}_{s-1}^{2}}{\max \left\{\mathfrak{I}_{s}^{2},\left[1+\mathfrak{J}_{s}^{2}\right] \mathfrak{J}_{1}^{2} /\left[1+\mathfrak{J}_{s}^{2}\right], \mathfrak{J}_{s}^{2} \cdot \mathfrak{I}_{1}^{2} / 1+\mathfrak{I}_{s}^{2}\right\}}=\frac{\mathfrak{I}_{s-1}^{2}}{\max \left\{\mathfrak{J}_{s}^{2}, \mathfrak{J}_{1}^{2}, \mathfrak{J}_{s}^{2} \cdot \mathfrak{I}_{1}^{2} / 1+\mathfrak{J}_{s}^{2}\right\}} \\
& =\frac{\mathfrak{J}_{s-1}^{2}}{\mathfrak{J}_{s}^{2}}=\frac{(s-1)^{2} s^{2}}{s^{2}(s+1)^{2}}=\frac{(s-1)^{2}}{(s+1)^{2}} \leq e^{-0.02} \text {. }
\end{aligned}
$$

Case 2. For each $s, t \in \mathbb{N}, s>t>1$, we get

$$
\begin{aligned}
& \frac{H\left(\varphi \mathfrak{I}_{s}, \varphi \mathfrak{I}_{t}\right)}{\beta\left(\mathfrak{I}_{s}, \mathfrak{J}_{t}\right)}=\frac{\left(\max \left\{\mathfrak{I}_{s-1}, \mathfrak{I}_{t-1}\right\}\right)^{2}}{\max \left\{\tau_{j}\left(\mathfrak{J}_{s}, \mathfrak{J}_{t}\right),\left[1+\tau_{j}\left(\mathfrak{J}_{s}, \varphi \mathfrak{I}_{s}\right)\right] \tau_{j}\left(\mathfrak{I}_{t}, \varphi \mathfrak{I}_{t}\right) / 1+\tau_{j}\left(\mathfrak{I}_{s}, \mathfrak{J}_{t}\right), \tau_{j}\left(\mathfrak{J}_{s}, \varphi \mathfrak{I}_{s}\right) \tau_{j}\left(\mathfrak{I}_{t}, \varphi \mathfrak{I}_{t}\right) / 1+\tau_{j}\left(\mathfrak{I}_{s}, \mathfrak{J}_{t}\right)\right\}} \\
& =\frac{\left(\max \left\{\mathfrak{J}_{s-1}, \mathfrak{J}_{t-1}\right\}\right)^{2}}{\max \left\{\tau_{j}\left(\mathfrak{J}_{s}, \mathfrak{J}_{t}\right),\left[1+\tau_{j}\left(\mathfrak{I}_{s}, \mathfrak{J}_{s-1}\right)\right] \tau_{j}\left(\mathfrak{I}_{t}, \mathfrak{J}_{t-1}\right) / 1+\tau_{j}\left(\mathfrak{J}_{s}, \mathfrak{J}_{t}\right), \tau_{j}\left(\mathfrak{J}_{s}, \mathfrak{J}_{s-1}\right) \tau_{j}\left(\mathfrak{J}_{t}, \mathfrak{J}_{t-1}\right) / 1+\tau_{j}\left(\mathfrak{J}_{s}, \mathfrak{J}_{t}\right)\right\}} \\
& =\frac{\mathfrak{I}_{s-1}^{2}}{\max \left\{\mathfrak{J}_{s}^{2},\left[1+\mathfrak{J}_{s}^{2}\right] \mathfrak{J}_{t}^{2}, /\left[1+\mathfrak{J}_{s}^{2}\right], \mathfrak{J}_{s}^{2} \cdot \mathfrak{J}_{t}^{2} / 1+\mathfrak{J}_{s}^{2}\right\}}=\frac{\mathfrak{I}_{s-1}^{2}}{\max \left\{\mathfrak{I}_{s}^{2}, \mathfrak{\Im}_{t}^{2}, \mathfrak{J}_{s}^{2} \cdot \mathfrak{J}_{t}^{2} / 1+\mathfrak{J}_{s}^{2}\right\}} \\
& =\frac{\mathfrak{\Im}_{s-1}^{2}}{\mathfrak{J}_{s}^{2}}=\frac{(s-1)^{2} s^{2}}{s^{2}(s+1)^{2}}=\frac{(s-1)^{2}}{(s+1)^{2}} \leq e^{-0.02} \text {. }
\end{aligned}
$$

Multiply 1.02 on the both cases and taking ln, we have

$$
\begin{aligned}
& \ln \left(1.02 H\left(\varphi \mathfrak{J}_{s}, \varphi \mathfrak{J}_{t}\right)\right)-\ln \left(\beta\left(\mathfrak{\Im}_{s}, \mathfrak{J}_{t}\right)\right) \\
& \leq \ln \left(1.02 \times e^{-0.02}\right) \Rightarrow \ln \left(1.02 H\left(\varphi \mathfrak{\Im}_{s}, \varphi \mathfrak{J}_{t}\right)\right)-\ln \left(\beta\left(\mathfrak{J}_{s}, \mathfrak{J}_{t}\right)\right) \\
& \leq-0.00019737 \Rightarrow \ln \left(\lambda H\left(\varphi \mathfrak{I}_{s}, \varphi \mathfrak{I}_{t}\right)\right)-\ln \left(\beta\left(\mathfrak{I}_{s}, \mathfrak{I}_{t}\right)\right) \\
& \leq-v \Rightarrow v+\Omega\left(\left(\lambda H \varphi \mathfrak{\Im}_{s}, \varphi \mathfrak{\Im}_{t}\right)\right) \leq \Omega\left(\beta\left(\mathfrak{\Im}_{s}, \mathfrak{\Im}_{t}\right)\right) .
\end{aligned}
$$

Therefore, the condition (iii) of Remark 9 is fulfilled with $v=0.00019737$ and $\lambda=1.02$. Hence, $\varphi$ has a unique FP.

\section{The Damped Harmonic Oscillator}

Assume that we have a body of mass $\mu$ moving back and forth on the $x$-axis around the equilibrium position $x=0$ and has a position $x(t)$ at time $t$ (see Figure 1). The force subjected to by the spring gives from

$$
F_{s}=-k x \text {. }
$$

In addition to the damping force that impedes the movement of the body, it gives from

$$
F_{d}=b \frac{d x}{d t}
$$

From the second law of motion we find that

$$
F_{\text {net }}=m \frac{d^{2} x}{d t^{2}}
$$

where,$b$, and $m$ are positive constants. Previously, this force is called the force damping oscillation.

Now suppose that there is an additional time-dependent force $F(t)$ that affects the body. Based on Newton's second law, we can write

$$
m \frac{d^{2} x}{d t^{2}}+b \frac{d x}{d t}+k x=F(t), x(0)=x^{\prime}(0)=0 .
$$

By Green's function, Problem (76) is equivalent to the following Fredholm integral equation:

$$
w\left(t_{1}\right)=\int_{0}^{1} O\left(t_{1}, r\right) B\left(t_{1}, r, w(r)\right) d r, t_{1} \in[0,1]
$$




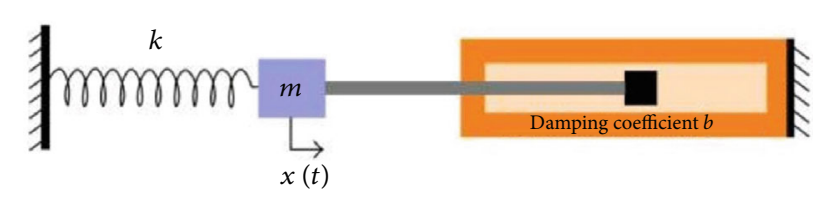

FIGURE 1: Forced oscillations.

where $B:[0,1] \times[0,1] \times \mathbb{R} \longrightarrow \mathbb{R}$ is a continuous function depends on $F$ and the continuous Green's function $O$ for critically damping oscillation is described by

$$
O\left(t_{1}, r\right)= \begin{cases}-r e^{v\left(t_{1}-t\right)}, & 0 \leq r \leq t_{1} \leq 1 \\ -t e^{v\left(t-t_{1}\right)}, & 0 \leq t_{1} \leq r \leq 1\end{cases}
$$

and $v$ can be found in terms of $m, b$, and $k$.

Suppose that $V=B(C[0,1], \mathbb{R})$ is the set of all bounded real continuous functions defined on $[0,1]$, equipped with

$$
\tau_{j}\left(z_{1}, z_{2}\right)=\left(\left\|z_{1}+z_{2}\right\|_{\infty}\right)^{2}, \forall z_{1}, z_{2} \in V,
$$

where $\|z\|_{\infty}=\sup _{t \in 0,1]}\left\{|z(t)| e^{-v}\right\}$. Define the function $j$ : $V \times V \longrightarrow[1, \infty)$ by $j\left(z_{1}, z_{2}\right)=1+\left|z_{1}\right|+\left|z_{2}\right|$. Then, $\left(V, \tau_{j}\right)$ is a complete $¥$-space.

In order to study the existence of a solution to Problem (77), we shall consider the following stipulations:

(a) There is a continuous function $B:[0,1] \times 0,1] \times$ $\mathbb{R} \longrightarrow \mathbb{R}$ and for $\left.r, t_{1} \in 0,1\right]$ and $\lambda>1$ so that

$$
\left|B\left(t_{1}, r, z_{1}(r)\right)+B\left(t_{1}, r, z_{1}(r)\right)\right|^{2} \leq \frac{e^{-v}}{\lambda} \beta\left(z_{1}(r), z_{2}(r)\right)
$$

for all $z_{1}, z_{2} \in \mathbb{R}$, where

$$
\beta\left(z_{1}, z_{2}\right)=\max \left\{\left|z_{1}+z_{2}\right|^{2}, \frac{\left[1+\left|z_{1}+\Phi z_{1}\right|^{2}\right]\left|z_{2}+\Phi z_{2}\right|^{2}}{1+\left|z_{1}+z_{2}\right|^{2}}, \frac{\left|z_{1}+\Phi z_{1}\right|^{2}\left|z_{2}+\Phi z_{2}\right|^{2}}{1+\left|z_{1}+z_{2}\right|^{2}}\right\}
$$

and $\Phi: C([0,1]) \longrightarrow ß C([0,1])$ is a multivalued operator defined on the beginning proof.

(b) $\int_{0}^{1} O\left(t_{1}, r\right) \leq 1$, for some $t_{1}>0$

Now, we present our main result in this part.

Theorem 11. Assume that $\left(V, \tau_{j}\right)$ is a complete $¥$-space. Then, Problem (77) has a unique solution provided that the stipulations (a) and (b) are fulfilled.
Proof. Define an operator $\Phi: C([0,1]) \longrightarrow ß C([0,1])$ by

$$
\Phi w\left(t_{1}\right)=\int_{0}^{1} O\left(t_{1}, r\right) B\left(t_{1}, r, w(r)\right) d r, t_{1} \in[0,1] .
$$

It should be noted that the unique FP of the mapping $\Phi$ is a solution of the nonlinear Problem (77). Hence, it is a solution to the differential equation of the second degree (76).

For $z_{1}, z_{2} \in V$, then by the hypotheses (a) and (b), we can get

$$
\begin{aligned}
\left(\left|\Phi z_{1}\left(t_{1}\right)+\Phi z_{2}\left(t_{1}\right)\right|\right)^{2} & =\left|\int_{0}^{1} O\left(t_{1}, r\right)\left[B\left(t_{1}, r, z_{1}(r)\right)+B\left(t_{1}, r, z_{2}(r)\right)\right] d r\right|^{2} \leq \int_{0}^{1}\left|O\left(t_{1}, r\right)\right|^{2}\left|B\left(t_{1}, r, z_{1}(r)\right)+B\left(t_{1}, r, z_{2}(r)\right)\right|^{2} d r \\
& \leq \int_{0}^{1}\left|B\left(t_{1}, r, z_{1}(r)\right)+B\left(t_{1}, r, z_{2}(r)\right)\right|^{2} d r \leq \int_{0}^{1} \frac{e^{-v}}{\lambda} \beta\left(z_{1}(r), z_{2}(r)\right) d r \\
& =\frac{e^{-v}}{\lambda} \int_{0}^{1} e^{v} \max \left\{\left|z_{1}+z_{2}\right|^{2} e^{-v}, \frac{\left[1+\left|z_{1}+\Phi z_{1}\right|^{2}\right]\left|z_{2}+\Phi z_{2}\right|^{2} e^{-2 v}}{1+\left|z_{1}+z_{2}\right|^{2} e^{-v}}, \frac{\left|z_{1}+\Phi z_{1}\right|^{2}\left|z_{2}+\Phi z_{2}\right|^{2} e^{-2 v}}{\left.1+\left|z_{1}+z_{2}\right|^{2} e^{-v}\right\} d r}\right. \\
& =\frac{e^{-v}}{\lambda} \int_{0}^{1} e^{r v} \max \left\{\tau_{j}\left(z_{1}, z_{2}\right), \frac{\left[1+\tau_{j}\left(z_{1}, \Phi z_{1}\right)\right] \tau_{j}\left(z_{2}, \varphi z_{2}\right)}{1+\tau_{j}\left(z_{1}, z_{2}\right)}, \frac{\tau_{j}\left(z_{1}, \Phi z_{1}\right) \tau_{j}\left(z_{2}, \varphi z_{2}\right)}{1+\tau_{j}\left(z_{1}, z_{2}\right)}\right\} d r \\
& =\frac{e^{-v}}{\lambda} \beta\left(z_{1}, z_{2}\right) \int_{0}^{1} e^{v} d r=\frac{e^{-v}}{\lambda} \beta\left(z_{1}, z_{2}\right) \times e^{v} .
\end{aligned}
$$


Hence for $z_{1}, z_{2} \in V$,

$$
\lambda\left(\left|\Phi z_{1}\left(t_{1}\right)+\Phi z_{2}\left(t_{1}\right)\right|\right)^{2} \times e^{-v} \leq e^{-v} \beta\left(z_{1}, z_{2}\right),
$$

which yields

$$
\lambda \tau_{j}\left(\Phi z_{1}, \Phi z_{2}\right) \leq e^{-v} \beta\left(z_{1}, z_{2}\right)
$$

Taking the log for the base $e$ to both side, we have

$$
\ln \left(\lambda \tau_{j}\left(\Phi z_{1}, \Phi z_{2}\right)\right) \leq-v+\ln \left(\beta\left(z_{1}, z_{2}\right)\right) .
$$

Describe the function $\Omega: \mathbb{R}^{+} \longrightarrow \mathbb{R}^{+}$by $\Omega(\sigma)=\ln (\sigma)$, $\sigma>0$ in (86), so that $\Omega \in \Sigma$, and we get

$$
v+\Omega\left(\lambda \tau_{j}\left(\Phi z_{1}, \Phi z_{2}\right)\right) \leq \Omega\left(\beta\left(z_{1}, z_{2}\right)\right)
$$

where

$$
\beta\left(z_{1}, z_{2}\right)=\max \left\{\tau_{j}\left(z_{1}, z_{2}\right), \frac{\left[1+\tau_{j}\left(z_{1}, \Phi z_{1}\right)\right] \tau_{j}\left(z_{2}, \Phi z_{2}\right)}{1+\tau_{j}\left(z_{1}, z_{2}\right)}, \frac{\tau_{j}\left(z_{1}, \Phi z_{1}\right) \tau_{j}\left(z_{2}, \Phi z_{2}\right)}{1+\tau_{j}\left(z_{1}, z_{2}\right)}\right\}
$$

Therefore, all requirements of Theorem 11 under a special case (iii) $\left(\Phi_{\Omega}^{r G S}\right.$-contraction) are fulfilled. Then, there is a unique FP of the mapping $\Phi$, which is a unique solution to Problem (77) and then to (76).

\section{Newton's Law of Cooling}

Newton's law of cooling is a differential equation that foresees the cooling of somebody that placed in a colder environment; then, the fixed point method can be applied in finding the solution of this differential equation as follows. Consider the IVP in the form of

$$
\mathfrak{R}^{\prime}(t)=\Xi(t, \mathfrak{R}(t)), \mathfrak{R}\left(t_{0}\right)=\mathfrak{R}_{0} .
$$
if [25]

We say that $\Re(t)$ is a solution of Problem (89) if and only

$$
\mathfrak{R}(t)=\mathfrak{R}_{0}+\int_{t_{0}}^{t} \Xi(\ell, \mathfrak{R}(\ell)) d \ell,
$$

for all $t, \ell \in\left[t_{0}, t\right]$, where the functions $\Re:\left[t_{0}, t\right] \longrightarrow \mathbb{R}$ and $\Phi:\left[t_{0}, t\right] \times \mathbb{R} \longrightarrow \mathbb{R}$ are continuous.

Define a distance norm $\tau_{j}(\wp, \hbar)=\left(\|\wp+\hbar\|_{\infty}\right)^{2}$ on the set of all real continuous functions $V=C\left(\left[t_{0}, t\right], \mathbb{R}\right)$ defined on $\left[t_{0}, t\right]$, for all $\wp, \hbar \in V$, where $\|\wp\|_{\infty}=\sup _{t \in\left[t_{0}, t\right]}\left\{|\wp(t)| e^{-t v}\right\}$. Then, the pair $\left(V, \tau_{j}\right)$ is a complete $¥$-space with $j(\wp, \hbar)=$ $1+|\wp|+|\hbar|$.

Now, we present the main theorem in this section.

Theorem 12. Assume that $\left(V, \tau_{j}\right)$ is a complete $¥$-space. Assume also, for all $t, \ell \in\left[t_{0}, t\right]$, there is a constant $v \in \mathbb{R}^{+}$so that

$$
|\Xi(\ell, \wp(\ell))+\Xi(\ell, \hbar(\ell))| \leq \frac{e^{-v / 2}}{\sqrt{\lambda}\left(t-t_{0}\right)}(|\wp(\ell)+\hbar(\ell)|),
$$

where $\wp, \hbar \in V, \lambda>1$. Let $\wp_{u}, \hbar_{u} \in V$ so that $\left|\wp_{u}+\hbar_{u}\right| \leq 1$, for all $0 \in \mathbb{R}$. Then, Equation (90) has a unique solution.
Proof. Define an operator $\Phi: V \longrightarrow V$ by

$$
\Phi \mathfrak{R}(t)=\mathfrak{R}_{0}+\int_{t_{0}}^{t} \Xi(\ell, \mathfrak{R}(\ell)) d \ell
$$

It is clear that, if $\Phi \Re=\Re$, then $\Re$ is a solution of the Equation (90) and then (89).

For $\wp, \hbar \in V$, one can write

$$
\begin{aligned}
& |\Phi \wp(t)+\Phi \hbar(t)|^{2} \\
& \quad=\left|\wp_{0}+\int_{t_{0}}^{t} \Xi(\ell, \wp(\ell)) d \ell+\hbar_{0}+\int_{t_{0}}^{t} \Xi(\ell, \hbar(\ell)) d \ell\right|^{2} \\
& \quad \leq\left(\int_{t_{0}}^{t}|\Xi(\ell, \wp(\ell))+\Xi(\ell, \hbar(\ell))| d \ell\right)^{2} \\
& \quad \leq\left(\int_{t_{0}}^{t} \frac{e^{-v / 2}}{\sqrt{\lambda}\left(t-t_{0}\right)}(|\wp(\ell)+\hbar(\ell)|) d \ell\right)^{2} \\
& =\left(\int_{t_{0}}^{t} \frac{e^{-v / 2}}{\sqrt{\lambda}\left(t-t_{0}\right)} \times \sqrt{e^{-2 v t} \times e^{2 v t}} \times(|\wp(\ell)+\hbar(\ell)|) d \ell\right)^{2} \\
& =\frac{e^{-v}}{\lambda\left(t-t_{0}\right)^{2}} \times \tau_{j}(\wp, \hbar) \times e^{2 v t}\left(\int_{t_{0}}^{t} d \ell\right)^{2} \\
& =\frac{e^{-v}}{\lambda} \tau_{j}(\wp, \hbar) e^{2 v t} \leq \frac{e^{-v}}{\lambda} \beta(\wp, \hbar) e^{2 v t},
\end{aligned}
$$

where

$\beta(\wp, \hbar)=\max \left\{\tau_{j}(\wp, \hbar), \frac{\left[1+\tau_{j}(\wp, \Phi \wp)\right] \tau_{j}(\hbar, \Phi \hbar)}{1+\tau_{j}(\wp, \hbar)}, \frac{\tau_{j}(\wp, \Phi \wp) \tau_{j}(\hbar, \Phi \hbar)}{1+\tau_{j}(\wp, \hbar)}\right\}$. 
Hence, one can write

$$
\left(|\Phi \wp(t)+\Phi \hbar(t)| \times e^{-v t}\right)^{2} \leq \frac{e^{-v}}{\lambda} \beta(\wp, \hbar),
$$

yields,

$$
\left(\|\Phi \wp(t)+\Phi \hbar(t)\|_{\infty}\right)^{2} \leq \frac{e^{-v}}{\lambda} \beta(\wp, \hbar) .
$$

It follows that

$$
\lambda \tau_{j}(\Phi \wp(t), \Phi \hbar(t)) \leq e^{-v} \beta(\wp, \hbar) .
$$

Consider $\Omega(\sigma)=\ln (\sigma)$ for $\sigma>0$ and $\Omega \in \Sigma$; then,

$$
\ln \left(\lambda \tau_{j}(\Phi \wp(t), \Phi \hbar(t))\right) \leq \ln \left(e^{-v} \beta(\wp, \hbar)\right),
$$

or

$$
v+\ln \left(\lambda \tau_{j}(\Phi \wp(t), \Phi \hbar(t))\right) \leq \ln (\beta(\wp, \hbar)) .
$$

Equivalently,

$$
v+\Omega\left(\lambda \tau_{j}(\Phi \wp(t), \Phi \hbar(t))\right) \leq \Omega(\beta(\wp, \hbar)) .
$$

Based on Theorem 8 under a special case (iii) of Remark 9 ( $\Phi_{\Omega}^{r G S}$-contraction), $\Phi$ possesses a unique $\mathrm{FP}$, which is a unique solution to Equation (90) and then to (89).

Now, we describe Newton's law of cooling as follows: Let

$$
\eta^{\prime}(t)=-\xi\left(\eta(t)-\eta_{\varkappa}\right)
$$

where $\eta(t)$ and $\eta_{\mathrm{u}}$ refer to the temperature of the object at the time $t$ and the temperature of the environment, respectively, and $\xi$ is proportionality constant. If we put $\eta\left(t_{0}\right)=\eta_{0}$, then we obtain the IVP below:

$$
\eta^{\prime}(t)=-\xi\left(\eta(t)-\eta_{\mathbf{u}}\right) \eta\left(t_{0}\right)=\eta_{0} .
$$

Assume that $\Xi(t, \eta)=-\xi\left(\eta(t)-\eta_{\mathrm{u}}\right)$. Then, we have

$$
\begin{aligned}
|\Xi(\ell, \wp(\ell))+\Xi(\ell, \hbar(\ell))| & =\left|-\xi\left(\wp(t)-\wp_{\varkappa}\right)-\xi\left(\hbar(t)-\hbar_{\varkappa}\right)\right| \\
& =\xi\left|\wp(\ell)+\hbar(\ell)-\left(\wp_{\mathrm{u}}+\hbar_{\varkappa}\right)\right| \\
& \leq \xi\left(|\wp(\ell)+\hbar(\ell)|+\left|\wp_{\varkappa}+\hbar_{\varkappa}\right|\right) \\
& \leq \xi|\wp(\ell)+\hbar(\ell)| \\
& =\frac{e^{-v / 2}}{\sqrt{\lambda}\left(t-t_{0}\right)}(|\wp(\ell)+\hbar(\ell)|) .
\end{aligned}
$$

According to Theorem 12, the initial value problem (102) has a unique solution. It should be noted that the exact solution of (102) is $\eta(t)=\eta_{\mathrm{u}}+\left(\eta_{0}-\eta_{\mathrm{u}}\right) e^{-\xi\left(t-t_{0}\right)}$.

\section{Infinite Systems of Fractional ODEs}

In this part, we study the sufficient condition to find the solution to an infinite nonlinear fractional system from ODEs on the space of all real sequences whose limit is finite which denoted with $\mathbf{c}$.

Assume that the system is defined as follows:

$$
\left\{\begin{array}{l}
\left.\left.\left.\neg_{\rho_{i}}=\frac{e^{-v}}{q(t)} q_{i}(t)\right\urcorner_{i}+\sigma_{i}(\neg,\urcorner_{1},\right\urcorner_{2}, \cdots\right), \rho_{i} \in(0,1), \\
\left.\left.\neg_{\kappa_{j}}=\frac{e^{-v}}{q(t)} q_{j}(t)\right\urcorner_{j}+\sigma_{j}(\neg,\urcorner_{1}, \neg_{2}, \cdots\right), \kappa_{j} \in(0,1), \\
\neg_{0}=\neg_{0}^{i}, i, j=1,2, \cdots,
\end{array}\right.
$$

where $t \in J, J$ is any fixed interval on the real line and $v$ is the positive real number.

Suppose that $V=\mathbf{c}$ equipped with $\tau_{j}(\wp, \hbar)=$ sup $\left\{\left|\wp_{i}+\hbar_{i}\right|^{2} e^{-v}\right\}$ for all $\wp, \hbar \in V$, and $j: V \times V \longrightarrow[1, \infty)$ is a function described by $j(\wp, \hbar)=1+|\wp|+|\hbar|$. Then, $\left(V, \tau_{j}\right)$ is a complete $¥$-space.

System (104) will be studied under the following hypotheses:

$\left(\right.$ hy $\left._{1}\right) \neg_{0}=\neg_{0}^{i} \in \mathbf{c}$ and $\sigma=\left(\sigma_{1}, \sigma_{2}, \cdots\right): J \times \mathbf{c} \longrightarrow \mathbf{c}$

$\left(\mathrm{hy}_{2}\right)$ the function $q_{i}(t)>0$ is a continuous for all $t \in J$ and $i=1,2 \cdots$

$\left(\right.$ hy $_{3}$ ) for all $\wp, \hbar \in V$, we have

$$
\left|\sigma_{i}(t, \wp)+\sigma_{i}(t, \hbar)\right| \leq \frac{e^{-v}}{2 \sqrt{\lambda}} \sqrt{\beta(\wp, \hbar)}, \lambda>1,
$$

where $\wp=\left(\wp_{1}, \wp_{2}, \cdots\right), \wp=\left(\hbar_{1}, \hbar_{2}, \cdots\right)$ and

$\beta(\wp, \hbar)=\max \left\{\tau_{j}(\wp, \hbar), \frac{\left[1+\tau_{j}\left(\wp, \Phi_{i} \wp\right)\right] \tau_{j}\left(\hbar, \varphi_{j} \hbar\right)}{1+\tau_{j}(\wp, \hbar)}, \frac{\tau_{j}\left(\wp, \Phi_{i} \wp\right) \tau_{j}\left(\hbar, \varphi_{j} \hbar\right)}{1+\tau_{j}(\wp, \hbar)}\right\}$,

(hy ${ }_{4}$ ) for all $\wp, \hbar \in V$ and for $t \in J$,

$$
\left|q_{i}(t) \wp+q_{j}(t) \hbar\right| \leq \frac{q(t)}{2 \sqrt{\lambda}} \sqrt{\beta(\wp, \hbar)} .
$$

Now, we state and prove our main theorem.

Theorem 13. System (104) has a unique solution under hypotheses $\left(h y_{1}\right)-\left(h y_{4}\right)$.

Proof. Let $\Phi_{i}(t, \wp), \varphi_{j}(t, \hbar): J \times \mathbf{c} \longrightarrow \mathbf{c}$ be mappings described by

$$
\left\{\begin{array}{l}
\Phi_{i}(t, \wp)=\frac{e^{-v}}{q(t)} q_{i}(t) \wp_{i}+\sigma_{i}\left(\wp_{,}, \wp_{1}, \wp_{2}, \cdots\right), \\
\varphi_{j}(t, \hbar)=\frac{e^{-v}}{q(t)} q_{j}(t) \hbar_{j}+\sigma_{j}\left(\hbar, \hbar_{1}, \hbar_{2}, \cdots\right) .
\end{array}\right.
$$


A sufficient condition for a solution to System (104) to exist is that there is a common FP of the mappings (108). To achieve this, consider

$$
\begin{aligned}
& \left|\Phi_{i}(t, \wp)+\varphi_{j}(t, \hbar)\right| \\
& \quad=\left|\frac{e^{-v}}{q(t)} q_{i}(t) \wp_{i}+\sigma_{i}\left(\wp_{1}, \wp_{2}, \cdots\right)+\frac{e^{-v}}{q(t)} q_{j}(t) \hbar_{j}+\sigma_{j}\left(\hbar, \hbar_{1}, \hbar_{2}, \cdots\right)\right| \\
& \quad \leq\left|\frac{e^{-v}}{q(t)} q_{i}(t) \wp_{i}+\frac{e^{-v}}{q(t)} q_{j}(t) \hbar_{j}\right|+\left|\sigma_{i}\left(\wp, \wp_{1}, \wp_{2}, \cdots\right)+\sigma_{j}\left(\hbar, \hbar_{1}, \hbar_{2}, \cdots\right)\right| \\
& \quad \leq \frac{e^{-v}}{q(t)} \times \frac{q(t)}{2 \sqrt{\lambda}} \sqrt{\beta(\wp, \hbar)}+\frac{e^{-v}}{2 \sqrt{\lambda}} \sqrt{\beta(\wp, \hbar)}=\frac{e^{-v}}{\sqrt{\lambda}} \sqrt{\beta(\wp, \hbar)} .
\end{aligned}
$$

Squaring both sides, we get

$$
\left|\Phi_{i}(t, \wp)+\varphi_{j}(t, \hbar)\right|^{2} \leq \frac{e^{-2 v}}{\lambda} \beta(\wp, \hbar),
$$

or

$$
\lambda\left|\Phi_{i}(t, \wp)+\varphi_{j}(t, \hbar)\right|^{2} e^{-v} \leq e^{-3 v} \beta(\wp, \hbar) \leq e^{-v} \beta(\wp, \hbar) .
$$

It follows that after taking the supremum,

$$
\lambda \tau_{j}\left(\Phi_{i}, \varphi_{j}\right) \leq e^{-v} \beta(\wp, \hbar)
$$

By taking the ln of both sides, we find that

$$
v+\ln \left(\lambda \tau_{j}\left(\Phi_{i}, \varphi_{j}\right)\right) \leq \ln (\beta(\wp, \hbar))
$$

Letting $\Omega(\sigma)=\ln (\sigma), \Omega \in \Sigma$, one can write

$$
v+\Omega\left(\lambda \tau_{j}\left(\Phi_{i}, \varphi_{j}\right)\right) \leq \Omega(\beta(\wp, \hbar))
$$

where

$\beta(\wp, \hbar)=\max \left\{\tau_{j}(\wp, \hbar), \frac{\left[1+\tau_{j}\left(\wp, \Phi_{i \wp)}\right) \tau_{j}\left(\hbar, \varphi_{j} \hbar\right)\right.}{1+\tau_{j}(\wp, \hbar)}, \frac{\tau_{j}\left(\wp, \Phi_{i} \wp\right) \tau_{j}\left(\hbar, \varphi_{j} \hbar\right)}{1+\tau_{j}(\wp, \hbar)}\right\}$.

Hence, we conclude by Theorem 12 that System (104) has a unique solution.

\section{Data Availability}

The data used to support the findings of this study are available from the corresponding author upon request

\section{Conflicts of Interest}

The authors declare that they have no competing interests concerning the publication of this article.

\section{Authors' Contributions}

All authors contributed equally and significantly in writing this article. All authors read and approved the final manuscript.

\section{Acknowledgments}

This work was supported in part by the Basque Government under Grant IT1207-19. The authors thank the Basque Government for Grant IT1207-19.

\section{References}

[1] S. Banach, "Sur les opérations dans les ensembles abstraits et leur application aux équations intégrales," Fundamenta Mathematicae, vol. 3, pp. 133-181, 1922.

[2] A. A. Kilbas, H. M. Srivastava, and J. J. Trujillo, "Theory and Applications of Fractional Differential Equations," in NorthHolland Mathematics Studies, vol. 204, Elsevier Science B.V, Amsterdam, 2006.

[3] S. G. Samko, A. A. Kilbas, and O. I. Marichev, Fractional Integrals and Derivatives, Translated from the 1987 Russian Original, Gordon and Breach, Yverdon, 1993.

[4] G. Wang, S. Liu, R. P. Agarwal, and L. Zhang, "Positive solutions of integral boundary value problem involving Riemann-Liouville fractional derivative," Journal of Fractional Calculus and Applications, vol. 4, no. 2, pp. 312-321, 2013.

[5] A. Atangana and D. Baleanu, "New fractional derivatives with nonlocal and non-singular kernel: theory and application to heat transfer model," Thermal Science, vol. 20, no. 2, pp. 763-769, 2016.

[6] T. Abdeljawad, R. P. Agarwal, E. Karapinar, and P. S. Kumari, "Solutions of the nonlinear integral equation and fractional differential equation using the technique of a fixed point with a numerical experiment in extended b-metric space," Symmetry, vol. 11, no. 5, p. 686, 2019.

[7] S. K. Panda, E. Karapınar, and A. Atangana, "A numerical schemes and comparisons for fixed point results with applications to the solutions of Volterra integral equations in dislocatedextendedb- metricspace," Alexandria Engineering Journal, vol. 59, no. 2, pp. 815-827, 2020.

[8] H. A. Hammad, H. Aydi, and N. Mlaiki, "Contributions of the fixed point technique to solve the $2 \mathrm{D}$ Volterra integral equations, Riemann-Liouville fractional integrals, and AtanganaBaleanu integral operators," Advances in Difference Equations, vol. 2021, no. 1, Article ID 3255, 2021.

[9] J. L. Lovoie, T. J. Osler, and R. Tremblay, "Fractional derivatives and special functions," SIAM Review, vol. 18, no. 2, pp. 240-268, 1976.

[10] A. Fernandez, "A complex analysis approach to AtanganaBaleanu fractional calculus," Mathematical Methods in the Applied Sciences, vol. 44, no. 10, pp. 8070-8087, 2021.

[11] F. Martnez, I. Martnez, M. K. A. Kaabar, R. Ortz-Munuera, and S. Paredes, "Note on the conformable fractional derivatives and integrals of complex-valued functions of a real variable," IAENG International Journal of Applied Mathematics, vol. 50, no. 3, pp. 609-615, 2020.

[12] S. B. Nadler, "Multi-valued contraction mappings," Pacific Journal of Mathematics, vol. 20, no. 2, pp. 475-488, 1969. 
[13] D. Wardowski, "Fixed points of a new type of contractive mappings in complete metric spaces," Fixed Point Theory and Applications, vol. 2012, no. 1, Article ID 2012, 2012.

[14] S. Czerwik, Nonlinear set-valued contraction mappings in bmetric spaces, vol. 46, no. 2, 1998Atti del Seminario Matematico e Fisico dell' Universita di Modena e Reggio Emilia, 1998.

[15] M. Alghamdi, N. Hussain, and P. Salimi, "Fixed point and coupled fixed point theorems on b-metric-like spaces," Journal of Inequalities and Applications, vol. 2013, Article ID 402, 2013.

[16] H. A. Hammad, M. De la Sen, and H. Aydi, “Analytical solution for differential and nonlinear integral equations via $F_{\omega_{e}}{ }^{-}$ Suzuki contractions in modified $\omega_{e}$-metric-like spaces," Journal of Function Spaces, vol. 2021, Article ID 6128586, 13 pages, 2021.

[17] M. Cosentino and P. Vetro, Fixed Point Results for F - Contractive Mappings of Hardy-Rogers-Type, vol. 28, Univerzitet u Nišu, 2014.

[18] M. Sgroi and C. Vetro, Multi-Valued F-Contractions and the Solution of Certain Functional and Integral Equations, vol. 27, Univerzitet u Nišu, 2013.

[19] M. Usman Ali, T. Kamran, and M. Postolache, "Solution of Volterra integral inclusion in $\mathrm{b}$ - metric spaces via new fixed point theorem," Nonlinear Analysis: Modeling and Control, vol. 2017, no. 1, pp. 17-30, 2017.

[20] H. A. Hammad and M. De la Sen, "Generalized contractive mappings and related results in b-metric-like spaces with an application," Symmetry, vol. 11, no. 5, p. 667, 2019.

[21] H. A. Hammad and M. de la Sen, "A coupled fixed point technique for solving coupled systems of functional and nonlinear integral equations," Mathematics, vol. 7, no. 7, p. 634, 2019.

[22] H. A. Hammad and M. De la Sen, "Solution of nonlinear integral equation via fixed point of cyclic $\alpha_{\mathrm{s}}^{\mathrm{q}}$ - rational contraction mappings in metric-like spaces," Bulletin of the Brazilian Mathematical Society, New Series, vol. 51, no. 1, pp. 81-105, 2020.

[23] B. Fisher, On a Theorem of Khan, vol. 4, Rivista di Matematica della Università di Parma, 1978.

[24] Z. Liu, B. Xu, and S. M. Kang, "Two fixed point theorems of mappings satisfying contractive inequalities of integral type," International Journal of Pure and Applied Mathematics, vol. 90, pp. 85-100, 2014.

[25] O. Plaat, Ordinary Differential Equations, Holden-Day, San Francisco, 1971 